\title{
Biomimetic Gelatin Methacrylate/Nano Fish Bone Hybrid Hydrogel for Bone Regeneration via Osteoimmunomodulation
}

\author{
Liping Huang, ${ }^{\dagger}$ Jianhua Zhang, ${ }^{\dagger}$ Junfei Hu, ${ }^{\star}$ Tianbao Zhao, ${ }^{* \dagger}$ and Zhipeng Gu** \\ $\uparrow$ School of Materials Science and Engineering, Xihua University, Chengdu 610039, PR. China \\ † College of Polymer Science and Engineering, Sichuan University, Chengdu 610065, PR. China \\ Email: zhaotb@ mail.xhu.edu.cn (T. Z.); guzhipeng2019@scu.edu.cn (Z.G.)
}

\section{Experimental Section \\ Preparation and characterization of NFB}

First, grass carp with meat was weighted, and then mixed with tap water at a mass ratio of 1:3 (fishbone: water). Then, the mixture was heated at $121^{\circ} \mathrm{C}$ for $1 \mathrm{~h}$ using a pressure cooker. After pressure cooking, the mixture was repeatedly washed with hot water (around $80^{\circ} \mathrm{C}$ ) for the removal of flesh, floating fat and other impurities, and then the white solid was collected. Next, the NFB was fabricated according to the previous report. ${ }^{1}$ In brief, the white solid was crushed into powders using a mincing machine, and then mixed with ice water at a mass ratio of 1:10. Subsequently, the mixture was micronized with a bone grinding machine at a speed of $2000 \mathrm{rpm}$, followed by the addition of equivalent $0.3 \%$ trilatone solution. The mixture solution was stimed at room temperature for $24 \mathrm{~h}$ with trilatone solution changed every $8 \mathrm{~h}$. After that, the mixture was thoroughly rinsed with a large amount of distilled water till the absence of bubbles and then decellularized bone mud was obtained. Next, the water content of decellularized bone mud was detected. Finally, bone mud with a water content of 5\% was poured into the wet high -energy ball mil and stirred at a speed of $3000 \mathrm{rpm}$ and under a ball mill filling rate of $85 \%$ and grinding ball diameter of $0.5 \mathrm{~mm}$. After $6 \mathrm{~h}$, nano fishbone solution was obtained and lyophilized for further reservation and utilization. Size distribution of the NFB was analyzed by dynamic light scattering (DLS, Malvern Zetasize NanoZS90, UK). The scanning electron microscope (SEM) was used to determine the morphology of the NFB. Zeta potential was also measured by DLS. The element composition of the NFB was analyzed by a field emis sion scanning electron micros cope equipped with a detector for energy dispersive X-ray spectroscopy and X-ray diffractometer (XRD).

\section{Fabrication of Gel-MA/NFB hydrogels}

The Gel-MA/NFB hydrogels were prepared by the photopolymerization of NFB and Gel-MA in an aqueous solution with an initiator. In brief, $10 \%(\mathrm{w} / \mathrm{v})$ freeze dried Gel-MA and 3\% (w/v) NFB were fully dissolved in distilled water with $1 \%(\mathrm{w} / \mathrm{v})$ Irgacure 2959 photoinitiator (Sigma-Aldrich). The mixture was then transferred to a custom-made 24-well mold (diameter 10 $\mathrm{mm}$ and thickness $5 \mathrm{~mm}$ for each well). Then, the mixture solution was irradiated with a long-wavelength UV lamp (Intell-ray 400 , Uvitron, $365 \mathrm{~nm}, 400 \mathrm{~W}$ ) for 1 2 min at room temperature at a 5 10 cm distance. The resultant hydrogels were removed from the mold and freeze dried in vacuumat $-50^{\circ} \mathrm{C}$ for 2 days prior to further characterization. All other preparation conditions were same.

\section{Characterization of hydrogels Interior morphology of hydrogels}

The porosity and interior morphology of Gel-MA/NFB hydrogels were researched by SEM. First, the swollen hydrogel samples were reached their maximum swelling ratio in distilled water at room temperature, quickly frozen in liquid nitrogen and 
then freeze-dried under vacuumat $-50^{\circ} \mathrm{C}$ for at least 2 days until all the solvent was removed. The freeze-dried hydrogel samples were then cut carefully and fixed on aluminum stubs and sputtered with gold for $30 \mathrm{~s}$ for interior morphology observation with a SEM instrument.

\section{Equilibrium swelling ratio and swelling kinetics of hydrogels}

Since biocompatibility apparently depends on water content, characterization of the amount of imbibed water in the swollen gel is important. ${ }^{2}$ The swelling kinetic of Gel-MA/NFB hydrogel was tested at $37^{\circ} \mathrm{C}$ over a period of $72 \mathrm{~h}$. The dry hydrogels were weighed before the test, and then they were immersed in $10 \mathrm{~mL}$ of PBS solution. Before weighing, the excess surface water of hydrogel samples was removed by filter papers at each measurement time point. The swelling ratio $\left(Q_{t}\right)$ of the hydrogels, at time $t$, is calculated as a function of time: $\mathrm{Q}_{\mathrm{t}}=\left[\left(\mathrm{W}_{\mathrm{t}}-\mathrm{W}_{0}\right) / \mathrm{W}_{0}\right] \times 100 \%\left(\mathrm{~W}_{\mathrm{t}}\right.$ : the weight of the hydrogel at time $\mathrm{t}, \mathrm{W}_{0}$ : the weight of the corres ponding dry hydrogel at $\mathrm{t}=0$ ). All swelling ratio results were obtained from triplicate samples and data were expressed as the mean \pm standard deviation.

\section{In vitro biodegradation of Gel-MA/NFB hydrogels}

In vitro biodegradation of the Gel-MA/NFB hybrid hydrogels was evaluated with phosphate buffer (PBS, PH=7.4). The dry hydrogels were weighed before the test, and then soaked in $10 \mathrm{~mL}$ of PBS buffer and incubated $24 \mathrm{~h}$ at $37^{\circ} \mathrm{C}$. At each test point, the hydrogels were removed and washed with distilled water for three times, follow by dried for $24 \mathrm{~h}$ at $37^{\circ} \mathrm{C}$ to remove the residual water and recorded the weight of hydrogels. The PBS buffer was refreshed every other day. The degradation percentage (DP) was determined by the following weight equation: $\mathrm{DP}=\left(\mathrm{W}_{0}-\mathrm{Wt}\right) / \mathrm{W}_{0} \times 100 \%$, where $\mathrm{W}_{0}$ represents the initial dry hydrogels weight and $\mathrm{Wt}$ is the weight at time $\mathrm{t}$. The DP was expressed as the mean $\pm \operatorname{standard}$ deviation $(\mathrm{n}=3)$.

\section{Rheological measurements}

The rheological properties of Gel-MA/NFB hydrogels were carried out by a rheometer (HAAKE, Thermo, Germany). A plateplate geometry with a diameter of $20 \mathrm{~mm}$ and plate-to-plate distance of $1 \mathrm{~mm}$ was used in all tests. At first, all hydrogel samples were fixed on the plate, and then the measurements of mechanical spectra were recorded in a constant strain mode, with a deformation of 0.05 maintained over the frequency range of $0.1-10 \mathrm{~Hz}(\mathrm{rad} / \mathrm{s})$ at $37^{\circ} \mathrm{C}$. The temperature dependence of Storage modulus $\left(\mathrm{G}^{\prime}\right)$ and loss modulus $\left(\mathrm{G}^{\prime \prime}\right)$ was measured with temperature scan ranging from 15 to $40^{\circ} \mathrm{C}$ (heating rate $1.75^{\circ} \mathrm{C} \mathrm{min}{ }^{-1}$ ) at a constant frequency of $1 \mathrm{~Hz}$ and a constant $\operatorname{strain}(\gamma=0.05,1.88 \mathrm{mrad})$.

\section{Cell culture}

In this study, the NIH 3T3 cells, hDPSCs cells, and RAW264.7 cells were used. The murine sourced macrophage cell line RAW264.7 cells were cultured in $\alpha$-MEM supplemented with $10 \%$ heat-inactivated fetal bovine serum and $1 \%$ (v/v) penicillin/streptomycin (P/S) in a humidified $\mathrm{CO}_{2}(5 \%)$ incubator with the temperature maintained at $37^{\circ} \mathrm{C}$. NIH $3 \mathrm{~T} 3$ cells were cultured in DMEM containing $10 \%$ fetal bovine serum(FBS) and $1 \%(\mathrm{v} / \mathrm{v})$ penicillin/streptomycin $(\mathrm{P} / \mathrm{S})$ at $37^{\circ} \mathrm{C}$ with a $5 \% \mathrm{CO}_{2}$ humidified atmos phere. RAW 264.7 cells were passaged by a cell scraper after reaching $80 \%$ confluence, while NIH 3T3 cells were pas saged by trypsinization. The hDPSCs utilized in the studies were collected and cultured as previously reported. ${ }^{3}$ Healthy human third molars were extracted from 12 adults (18-20 years of age; 6 males and 6 females) at the Department of Oral and Maxillofacial Surgery, the Affiliated Stomatological Hospital of Sun Yat-sen University. Informed consent was obtained from all the participants, and approval was granted by the Sun Yat-sen University Research Ethics Committee. Briefly, pulp tissues were is olated from the teeth and diges ted with type I collagenase $(3 \mathrm{mg} / \mathrm{mL}$ ) and $4 \mathrm{mg} / \mathrm{mL}$ dis pase (Gibco-BRL, Grand Is land, $\mathrm{NY}$, USA) at $37^{\circ} \mathrm{C}$ for $30 \mathrm{~min}$. After that, the cells were cultured in $\alpha$-MEM (Gibco-BRL) supplemented with $10 \%$ of fetal calf serum(Gibco-BRL), $100 \mu \mathrm{g} / \mathrm{mL}$ streptomycin, $100 \mathrm{U} / \mathrm{mL}$ penicillin (HyClone, Logan, UT, USA), and 5 mmo1/L glutamines (Gibco-BRL) at $37^{\circ} \mathrm{C}$ and $5 \% \mathrm{CO}_{2}$. All the hDPSCs is olated from 12 adults were mixed together for all the experiments .

\section{Proliferation and attachment of NIH 3T3 on Gel-MA /NFB hydrogels}


The live/dead cell staining as say and methyl thiazolyl tetrazolium(MTT) viability assay were used to research the in vitro cytotoxicity of the hydrogels. For the live/dead staining, the Gel-MA/NFB hydrogels were cut into round shapes with a diameter that just filled the well of a 24 -well cell culture plate and immersed into the $75 \%$ alcohol for $4 \mathrm{~h}$, then sterilized under UV light (in the DMEM) for at least $2 \mathrm{~h}$ before being put into the 24-well cell culture plates. After that the hydrogels were washed twice by PBS buffer and DMEM. Then, the hydrogels were placed in the 24 -well plate and the cells were seeded at a density of $1 \times 10^{4}$ cells perwell and incubated at $37^{\circ} \mathrm{C}$ for $30 \mathrm{~min}$ (to prevent cells from slipping away from hydrogel to the culture dish) and then added the medium. At day 1, 3 and 5, cells were stained with $1 \mu \mathrm{g} / \mathrm{mL}$ Calcein-AM and PI(Propidium iodide, Sigma-Aldrich, 1 $\mu \mathrm{g} / \mathrm{mL}$ ) at room temperature for $30 \mathrm{~min}$ to label the living and the dead cells, respectively. After that the cell vitality on the hydrogel surface was imaged by a fluorescence microscope. MTT [3-(4, 5-dimethylthiazol-2-yl)-2, 5-diphenyl tetrazolium bromide] as say was used to evaluate effect of Gel-MA/NFB hydrogels extracts on the proliferation of NIH 3T3 cells. NIH 3T3 cells were seeded in a density of $1 \times 10^{3}$ cells per well (96 well plate) and cultured overnight at $37^{\circ} \mathrm{C}$ under $5 \% \mathrm{CO}_{2}$. The culture medium was subsequently removed and replaced by material extract medium. At days 1, 3 and 5, $20 \mu \mathrm{L}$ of MTT solution (5 $\mathrm{mg} / \mathrm{mL}$ in PBS) was added into each well. The MTT medium solution was removed after $4 \mathrm{~h}$ incubation and $150 \mu \mathrm{L}$ of dimethyl sulfoxide (DMSO) was added to dis solve the formazan crystals. After being vortexed for 8 min, quantitative detection of the samples was done on a microplate reader to measure the absorbance at the wavelength of $490 \mathrm{~nm}$.

\section{Proliferation of RAW264.7 cells with Gel-MA /NFB hydrogels}

MTT as say was performed to evaluate the proliferation of RAW 264.7 cells with Gel-MA/NFB hydrogels extracts. RAW264.7 cells were seeded in a density of $10^{4}$ cells per well (96 well plate) and cultured overnight. The culture medium was subsequently removed and replaced by material extract medium. At day 1 and 2, $20 \mu \mathrm{L}$ of MTT solution $(5 \mathrm{mg} / \mathrm{mL}$ in PBS) was added to the medium in each well. The MTT/medium solution was removed after $4 \mathrm{~h}$ and $150 \mu \mathrm{L}$ of DMSO was added to dissolve the formazan crystals. Quantitative detection was done on a microplate reader to measure the absorbance at the wavelength of $490 \mathrm{~nm}$.

\section{Osteoimmunomodulatory effects of Gel-MA/NFB hydrogels on macrophages RAW264.7 cell morphology on NFB or Gel-MA/NFB hydrogels}

RAW 264.7 cells were seeded in 6 well plates at a density of $1.5 \times 10^{5}$ cells per well. After $24 \mathrm{~h}$, the culture medium was removed and replaced by $3 \mathrm{~mL}$ of $\alpha$-MEM (with $10 \%$ heat-inactivated fetal bovine serum) containing NFB particles in different concentrations $(0,200,300,500$ and $1000 \mu \mathrm{g} / \mathrm{mL})$ or Gel-MA/NFB hydrogels. The cells were incubated for $24 \mathrm{~h}$, the medium was removed and the cells were washed with PBS three times and then fixed with $4 \%$ paraformaldehyde at roomtemperature for $20 \mathrm{~min}$, after that, the cells were permeabilized using 1\% Triton X-100 for 5 10 min, and then were washed with PBS twice. Then, the cells were incubated with Rhodamine phalloidin $(5 \mu \mathrm{g} / \mathrm{mL})$ for 30 min to stain the cytoskeletons and were was hed with PBS twice. In the end, 4',6-diamidino-2-phenylindole (DAPI, $5 \mu \mathrm{g} / \mathrm{mL}$ ) was added to stain the nucleifor $5 \sim 10$ min. Images were captured by a Fluorescence microscope.

\section{TRAP staining of RAW264.7 cells}

Tartrate-resistant acid phosphatase (TRAP) is a metalloenzyme highly expressed in activated macrophages and osteoclast cells. This acid phosphatase can be detected by naphthol AS-Bi phosphoric acid in conjunction with diazonium salts. Therefore, the effect of Gel-MA/NFB hydrogels on the differentiation and maturation of RAW264.7 macrophages into multinucleated osteoclasts was investigated. The RAW 264.7 cells were plated at a density of $1 \times 10^{4}$ cells/well in 48 -well plates for $24 \mathrm{~h}$ and then incubated with $\alpha$-MEM (containing 10\% FBS, 1\% P/S, Gel-MA/NFB hydrogels) for 3 days to stimulate RAW264.7 cells into macrophage-like cells. TRAP staining was performed to on days 3 after the induction of macrophage phenotype, following the protocol(BestBio, China). TRAP buffer without tartrate solution was applied as control. After staining, the 48-well plates were photographed by a Nikon digital camera. The TRAP-positive cells with three or more nuclei (multinucleate cells) were counted from multiple image frames and statis tically analyzed. 


\section{Flow cytometry}

Expres sion of macrophage surface markers CCR7 (M1 marker) and CD163 (M2 marker) were detected by flow cytometry. After 3 days of stimu lation by material, the cells were physically detached and centrifuged. The obtained cells were washed with PBS. Samples were then incubated with CCR7 antibodies (Biolegend, PE) or CD163 antibodies (Biolegend, PE) at $37^{\circ} \mathrm{C}$ for 30 45 min to labelCCR7 or CD163 respectively. Theblank group incubated with no antibodies. The treated cells were analyzed by flow cytometer and data analysis were performed using Flowing Software.

\section{Inflammatory response of macrophages on Gel-MA/NFB hydrogels}

RAW 264.7 cells were seeded on different Gel-MA/NFB hydrogels in 6 well plate at a density of $1 \times 10^{5}$ cells per well and incubated for $4 \mathrm{~d}$. Then, the culture medium of macrophages was collected to examine the expression of Tumor necrosis factor $\alpha(\mathrm{TNF}-\alpha)$, Interleukin $1 \beta$ (IL-1 $\beta$ ), Interleukin 6 (IL-6), Oncostatin M (OSM) and Interleukin 10 (IL-10) by ELISA kits (CUSABIO, China). Gene expres sion of inflammatory cytokine (TNF- $\alpha$, IL-18, IL-1 $\beta$, OSM and IL-6) was as sessed by reversetranscription quantitative PCR (RT-qPCR). After culture medium collection, the culture plates were washed with PBS and then the total RNA was extracted by TRIZOL reagent. Complementary DNA (cDNA) was synthesized from $2 \mu \mathrm{g}$ of total RNA using GoScript ${ }^{\mathrm{TM}}$ Reverse Transcription System. RT-PCR was performed to quantify gene expression levels by means of the SYBR Premix Ex Taq (Takara). The primers used in this section is shown in Table S1. GADPH was used as a housekeeping gene. RAW264.7 cells cultured with complete medium served as the control groups.

\section{Osteogenic differentiation evaluation of macrophage-conditioned medium}

\section{The mineralization of $h D P S C s$}

To research the effect of the Gel-MA/NFB hydrogel-stimulated macrophage on the osteogenic differentiation of hDPSCs. Conditioned medium was prepared by culturing RAW264.7 cells $\left(1 \times 10^{5}\right.$ cells/well, 6 -well plate $)$ on Gel-MA or Gel-MA/NFB hydrogels for $4 \mathrm{~d}$, and then the supernatants were collected and mixed with complete DMEM medium(10\% FBS, 1\% P/S) at a ratio of 1:2 (labeled as condition medium) for the following cell culture. Alizarin Red staining was used to identify mineralization nodule. The hDPSCs cells were seeded on the hydrogels in 24-well plates at a density of $1 \times 10^{4}$ cells per well containing conditioned medium or complete medium. After stimulation for $6 \mathrm{~d}$, the condition medium was replaced by osteogenesis induction medium (OIM) (DMEM with 10\% FBS, 1\% P/S, $10 \mathrm{mM}$ b-glycerophosphate, $50 \mu \mathrm{g} / \mathrm{ml}$ ascorbic acid, and 10nM dexamethasone) and was refreshed every two days. After 4, 7, 14 days, the OIM was removed and the cells were washed with PBS, follow by fixation by in $4 \%$ paraformaldehyde for $20 \mathrm{~min}$. After being rinsed with PBS three times, the cells were stained with a solution containing $2 \%$ Alizarin Red S at pH(4.1 4.3, Sigma-Aldrich) for $30 \mathrm{~min}$. Then, the cells were washed with PBS four times before images acquired with a light microscope. The groups without conditioned media and samples were used as the blank group. Quantitative results of the alizarin red staining were analyzed by image J. The groups without materials were used as the control group. The Gel-MA group treated with condition medium and Gel-MA hydrogel. The Gel-MA/1\%NFB group treated with Gel-MA/1\%NFB hydrogel and condition medium. The Gel-MA/3\%NFB group treated with Gel-MA/3\%NFB hydrogel and condition medium. The Gel-MA/5\%NFB group treated with Gel-MA/5\%NFB hydrogel and condition medium.

\section{Alkaline phosphatase activity of hDPSCs}

Alkaline phosphatase activity of hDPSCs was used to access the effect of the Gel-MA/NFB hydrogels-stimulated macrophage on the os teogenic differentiation of hDPSCs. hDPSCs cells were seeded on the hydrogels in 24 -well plate at a density $1 \times 10^{4}$ cells per well with conditioned medium or complete medium. After culture for 6 days, the conditioned medium was removed and replaced by OIM. The ALP activity as say was performed after differentiation 4, 7, 14 days of induction. Steps are as follows, the medium was removed and the cells were washed with PBS. Then, the cells were detached fromthe hydrogels by trypsinization, followed by centrifugation for $5 \mathrm{~min}$ at $1200 \mathrm{rpm}$. Next, $1 \%$ Triton X-100 with PMSF $(150 \mu \mathrm{L})$ was added to lyse the cells for $30 \mathrm{~min}$ and the mixture was centrifuged at $12000 \mathrm{rpm}$ for $5 \sim 10 \mathrm{~min}$ at $4^{\circ} \mathrm{C}$. $50 \mu \mathrm{L}$ of working solution was mixed with $50 \mu \mathrm{L}$ supernatant samples, which was subsequently evaluated by the Alkaline Phosphatase Assay Kit (Beyotime Biotechnology) 
following the manufacturer's instructions. The ALP activity was calculated as the changed optical density (OD) values. The groups without conditioned media and samples were used as the blank group. The groups without materials were used as the control group. The Gel-MA group was treated with condition mediumand Gel-MA hydrogel. The Gel-MA/1\%NFB group was treated with Gel-MA/1\%NFB hydrogel and condition medium. The Gel-MA/3\%NFB group was treated with Gel-MA/3\%NFB hydrogel and condition medium. The Gel-MA/5\%NFB group was treated with Gel-MA/5\%NFB hydrogel and condition medium

\section{Osteogenesis-related genes and protein Expression}

The expres sion of osteogenesis-related genes and proteins was also detected by RT-qPCR and ELISA kits to evaluate the effect of the Gel-MA/NFB hydrogels-stimulated macrophage on the osteogenic differentiation of hDPSCs. The hDPSCs cells were seeded on the hydrogels in 6-well plates at a density $2 \times 10^{4}$ cells per well with conditioned mediumor complete medium. After culture for 6 days, the conditioned medium was removed and replaced by OIM. After 4, 7, 14 days, the culture medium was centrifuged (1200 rpm for $2 \mathrm{~min}$ ) to collect supernatants and examine the concentrations of osteogenesis-related proteins and angiogenesis-related proteins containing type I collagen (COL-1), human vascular endothelial growth factor (VEGF) and runtrelated transcription factor 2 (RUNX-2) by ELISA kits (CUSABIO, China) according to the manufacturer's instructions. The RT-qPCR was employed to evaluate the mRNA expression of osteogenic genes. The total RNA of the cultured hDPSCs was extracted by a TRIZOL reagent (Invitrogen). Complementary DNA (cDNA) was synthesized from $2 \mu \mathrm{g}$ of total RNA using GoScript ${ }^{\mathrm{TM}}$ Reverse Transcription System. RT-PCR was performed to quantify gene expression levels by means of the SYBR Premix Ex Taq (Takara). Expression levels of the osteogenesis-related genes including COL-1, VEGF, RUNX-2, and Osteopontin (OPN) was quantified. GADPH was used as the housekeeping gene. The forward and reverse primers of the selected genes were listed in Table S1. The groups without conditioned media and samples were used as the blank group. The groups without materials were used as the control group. The Gel-MA group was treated with condition mediumand Gel-MA hydrogel. The Gel-MA/1\%NFB group was treated with Gel-MA/1\%NFB hydrogel and condition medium. The Gel-MA/3\%NFB group was treated with Gel-MA/3\%NFB hydrogel and condition medium. The Gel-MA/5\%NFB group was treated with GelMA/5\%NFB hydrogel and condition medium.

\section{Rat model of bone defects}

All animal procedures were approved by the Animal Care and Use Committee of Sun Yat-sen University (GuangZhou, China). Sprague-Dawley rats (250 280 g, 4 weeks old) were anesthetized by abdominal cavity injection using the chloral hydrate (30 $\mathrm{mg} / \mathrm{kg}$ ). A midline incision was made to raise a full-thicknes s flap and expose the parietal bone. Two full-thickness craniotomies defects ( $5 \mathrm{~mm}$ diameter) were created by using a bone trephine drill (Traus 90) with the irrigation of sterile saline to preven theat injuries. A cylinder with a diameter of $5 \mathrm{~mm}$ and a thicknes s of $1 \mathrm{~mm}$ Gel-MA/NFB hydrogel or Gel-MA hydrogel was placed into the clean defect site of the animals. Finally, the incision was closed with sutures. The animals were sacrificed 4 weeks' postsurgery and the bone tis sues were collected and immersed in $4 \%$ buffered paraformaldehyde. A microcomputed tomography (micro-CT) system (Skyscan 1172) was applied to examine the samples and the volume of the newly formed bone (BV/TV), bone mineral density (BMD) were determined by the auxiliary histomorphometric software (CTAn).

\section{Histological and immunohistochemical analysis}

For histological analysis, the bone tis sues were decalcified in $10 \%$ ethylenediaminetetraacetic (EDTA) acid for one month, dehydrated in a graded series of alcohol, and finally embedded in paraffin. The specimens were cut at a $4 \sim 5 \mu \mathrm{m}$ thickness in the sagittal direction along the artificial defect and the cranial bone. Histological observation was performed with hematoxylin and eosin (H\&E) and Masson staining (Servicebio). Semi-qualification of the new bone formation was performed by analyzing H\&E images by using Image J. Additionally, immunohis tochemical as sessment was used to evaluate the expres sion of COL-1, VEGF, and OPN and inflammatory cytokines (TNF- $\alpha, \mathrm{IL}-6, \mathrm{IL}-1 \beta, \mathrm{IL}-10)$, this further confirmed the immune reaction caused by GelMA/NFB hydrogels. 


\section{Statistical Analysis}

All statistical computations were performed using SPSS software, and the statistical significance was analyzed using one-way ANOVA. All the data are shown as means \pm standard deviation, and the levelof significance was set at $\mathrm{P}<0.05$. 


\section{Tables and Figures Captions}

Table S1 Primer pairs used in the RT-qPCR.

\begin{tabular}{|c|c|c|}
\hline Gene & Forward primer $\left(5^{\prime}-3{ }^{\prime}\right)$ & Reverse primer (5'-3') \\
\hline TNF- $\alpha$ & AGCCGATGGGTTGTACCTG & ATAGCAAATCGGCTGACGGT \\
\hline IL-6 & TGTTCTCTGGGAAATCGTG & CAAGTGCATCATCGTTGTTCATAC \\
\hline IL- $1 \beta$ & ATGCCACCTTTTGACAGTGATG & TGATGTGCTGCTGCGAGATT \\
\hline OSM & ATGCAGACACGGCTTCTAAGA & TTGGAGCAGCCACGATTGG \\
\hline GADPH & AACTTTGGCATTGTGGAAGG & GGATGCAGGGATGATGTTCT \\
\hline IL-18 & GACTCTTGCGTCAACTTCAAGG & CAGGCTGTCTTTTGTCAACGA \\
\hline RUNX-2 & GGTACTTCGTCAGCATCCTATCA & GTCAGCGTCAACACCATCATT \\
\hline OPN & TGACCAGAGTGCTGAAACCCA & TTCCTGACTATCAATCACATCGG \\
\hline COL-I & ACGAAGACATCCCACCAATCA & CAGATCACGTCATCGCACAAC \\
\hline VEGF & GGCGGCCTTCGCTTACTC & GGCTGCTTCTTCCAACAATG \\
\hline
\end{tabular}


Table S2 Atomic proportion (\%) in NFB particles.

\begin{tabular}{|c|c|c|}
\hline Element & $\mathrm{Wt} \%$ & $\mathrm{Wt} \%$ Sigma \\
\hline $\mathrm{C}$ & 0.15 & 0.00 \\
\hline $\mathrm{O}$ & 71.04 & 0.26 \\
\hline $\mathrm{Na}$ & 0.44 & 0.12 \\
\hline $\mathrm{Mg}$ & 0.80 & 0.09 \\
\hline $\mathrm{Al}$ & 0.19 & 0.07 \\
\hline $\mathrm{P}$ & 8.19 & 0.10 \\
\hline $\mathrm{K}$ & 0.00 & 0.05 \\
\hline $\mathrm{Ca}$ & 19.21 & 0.19 \\
\hline Total: & 100.00 & \\
\hline
\end{tabular}




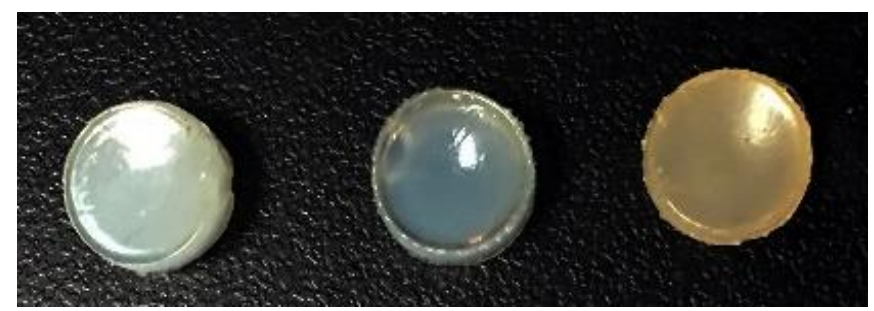

Figure S1. Images of Gel-M A hy drogels and Gel-M A/NFB hydrogels.

(a)

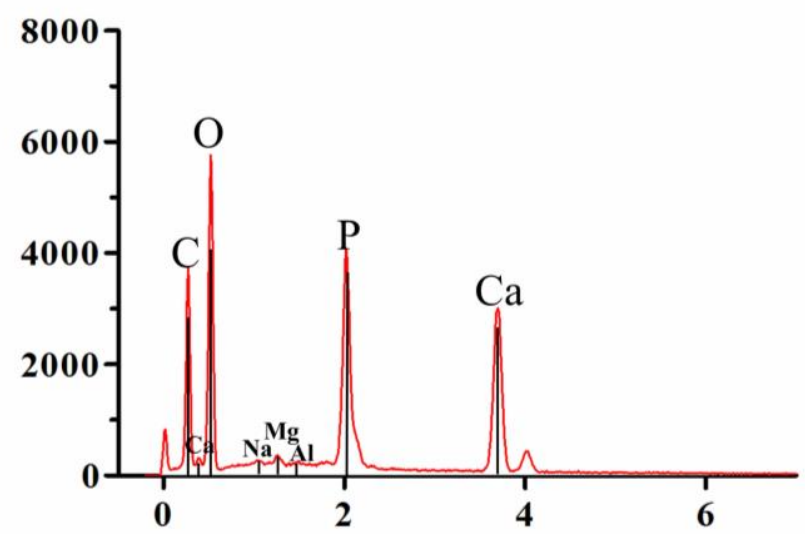

(b)

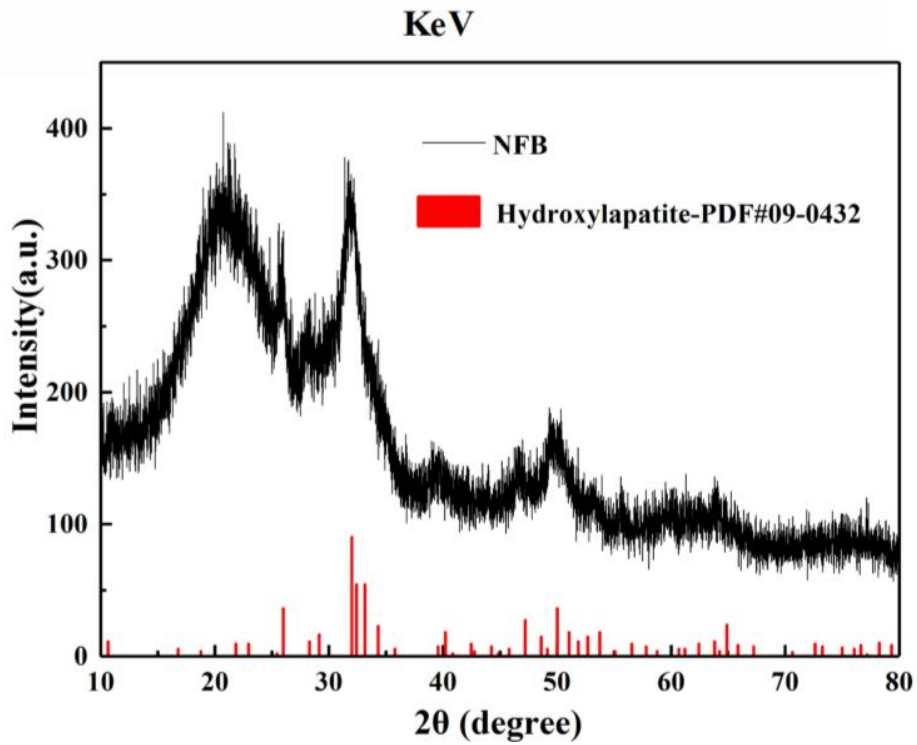

Figure S2. (a) EDS spectra of nano fish bone (NFB); (b) XRD spectra of NFB. 
(a)

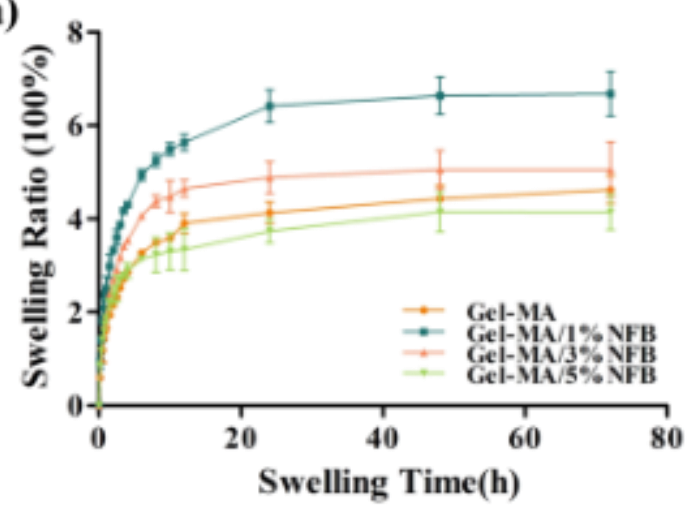

(c)

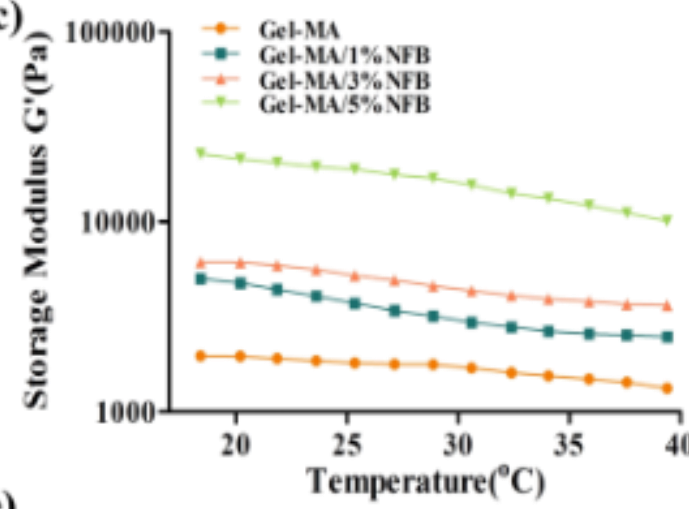

(e)

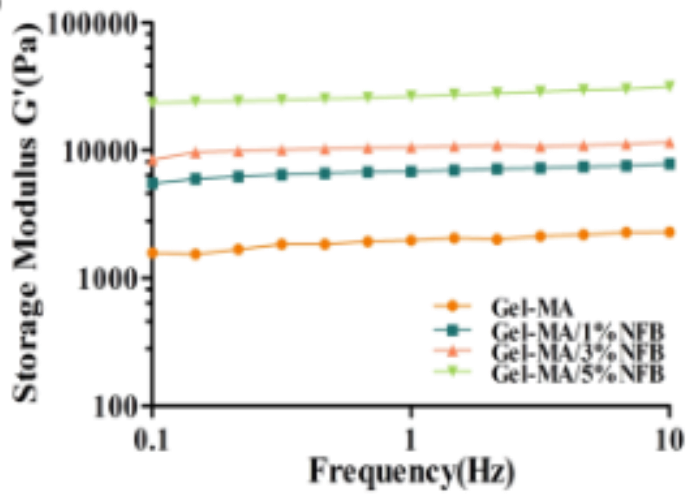

(b)
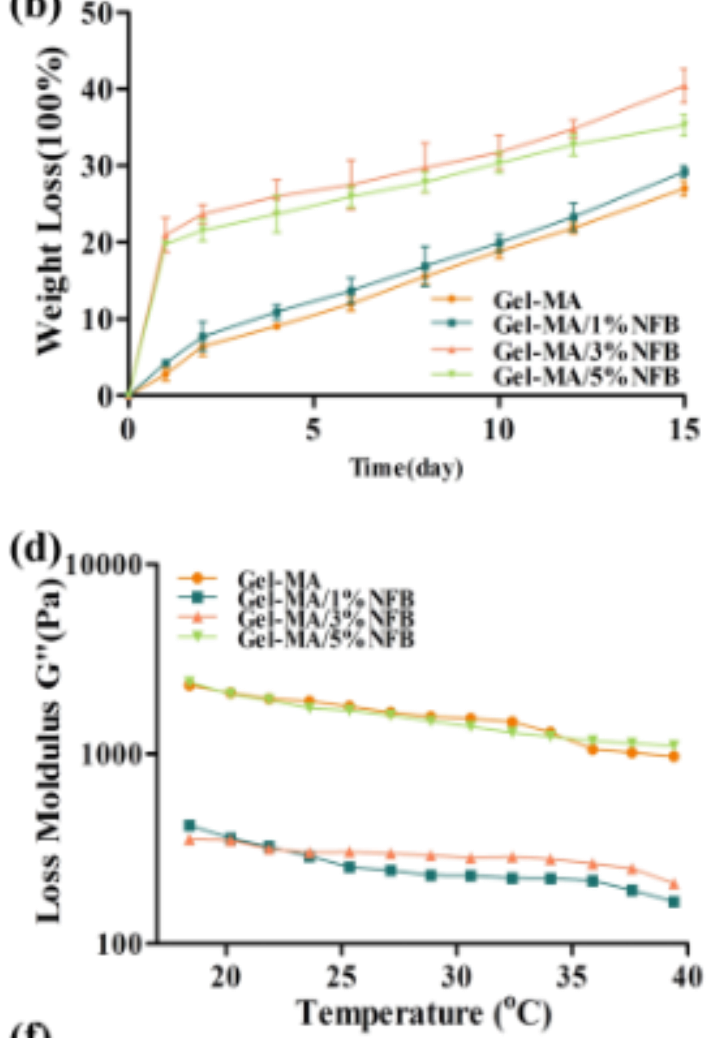

(f)

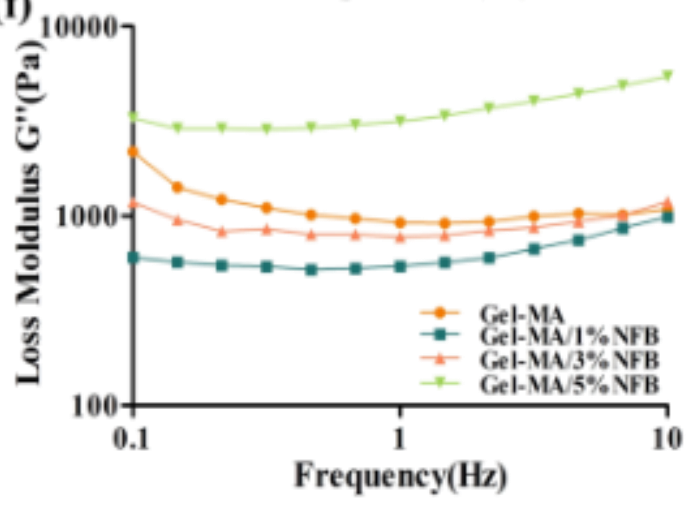

Figure S3. Characterization of Gel-M A/NFB hydrogels. (a) Swelling kinetics of the Gel-M A hy drogels and Gel-M A/NFB hy drogels in PBS at $37^{\circ} \mathrm{C}$; (b) In vitro biodegradation of Gel-M A/NFB hydrogels in PBS at $37^{\circ} \mathrm{C}$; (c, d) Effect of frequency on storage modulus $\left(\mathrm{G}^{\prime}\right)$ and loss modulus $\left(\mathrm{G}^{\prime \prime}\right)$ with Gel-MA hydrogels and Gel-MA/NFB hydrogels; (e, f) Effect of temperatures on storage modulus $\left(\mathrm{G}^{\prime}\right)$ and loss modulus $\left(\mathrm{G}^{\prime \prime}\right)$ with Gel-M A hy drogels and Gel-M A/NFB hy drogels. 

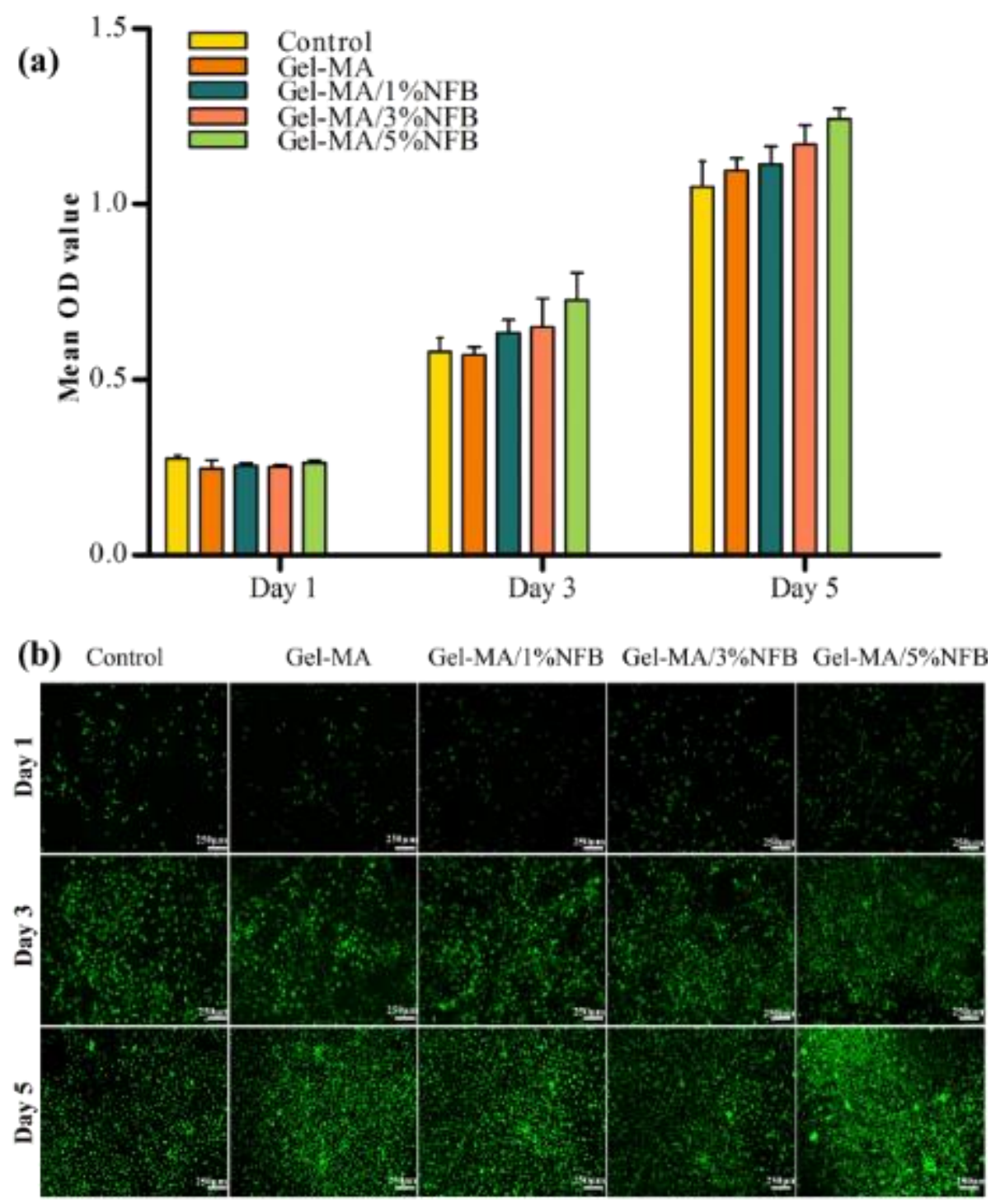

Figure S4. A cy tocompatibility assay performed on hydrogels. (a) MTT assay; (b) live/dead staining assay to evaluate the viability of NIH 3 T3 cultured with a Gel-MA hy drogel or a Gel-M A/NFB hy drogel.

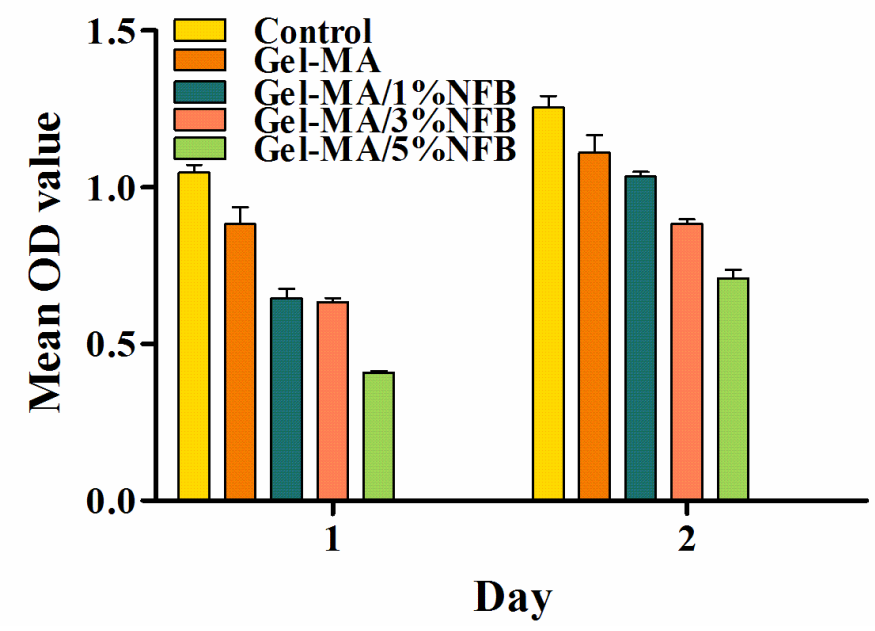

Figure S5. The viability of macrophages (RAW264.7 cells) by MTT analy sis cultured with Gel-MA/NFB hydrogels extracts. 


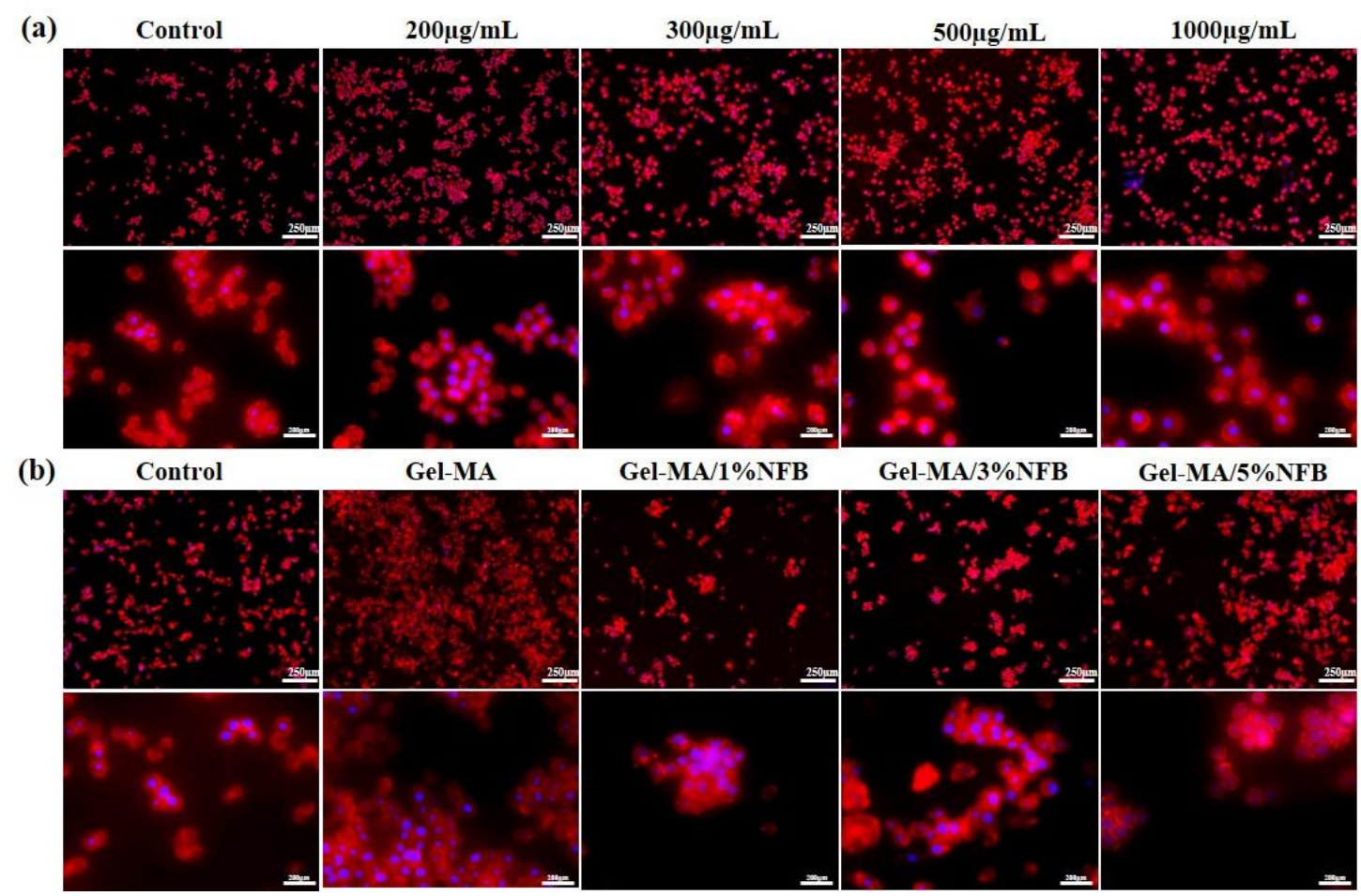

Figure S6. Inflammatory response of macrophages cultured on Gel-M A hy drogels or Gel-M A/NFB hy drogels. (a) The cell morphology of macrophages on NFB was stained with DAPI (blue, nuclei) and Rhodamine phalloidin (red, cytoskeleton); (b) The cell morphology of macrophages on Gel-M A/NFB hy drogels was stained with DAPI and Rhodamine phalloidin.

(a)

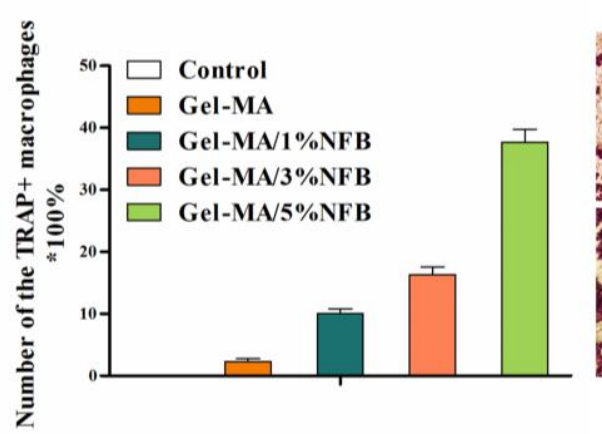

(b)

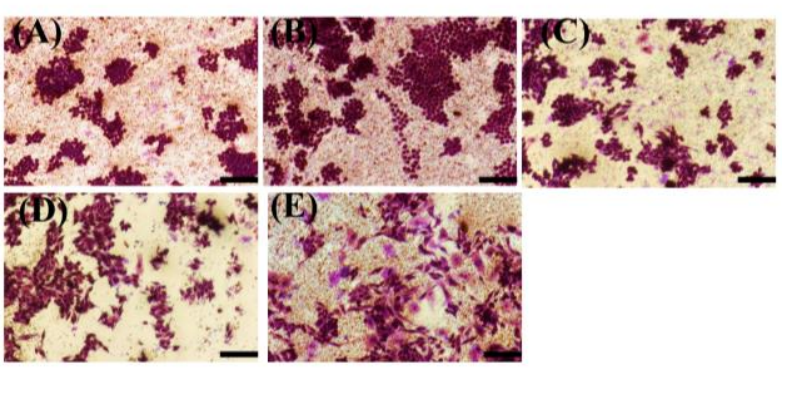

Figure S7. (a) Quantitatively analy sis the percentage of TRAP-positive cells; (b) Typical TRAP staining images of RAW264.7 monocy tes cultured on Gel-M A hy drogels or Gel-M A/NFB hy drogels. Scale bar = $100 \mu \mathrm{m}$; (A) control; (B) Gel-M A; (C) GelMA/1\%NFB; (D) Gel-M A/3\%NFB; (E) Gel-M A/5\%NFB. 


\section{CD163}

(a)

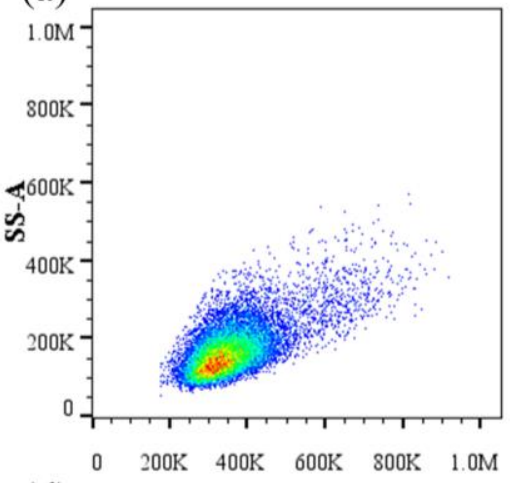

(d)

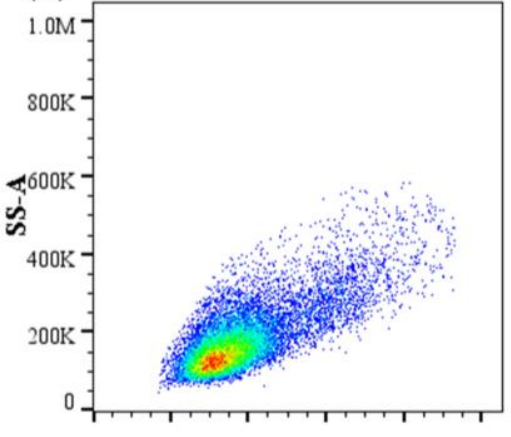

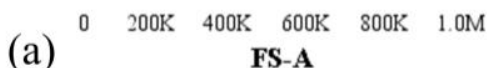

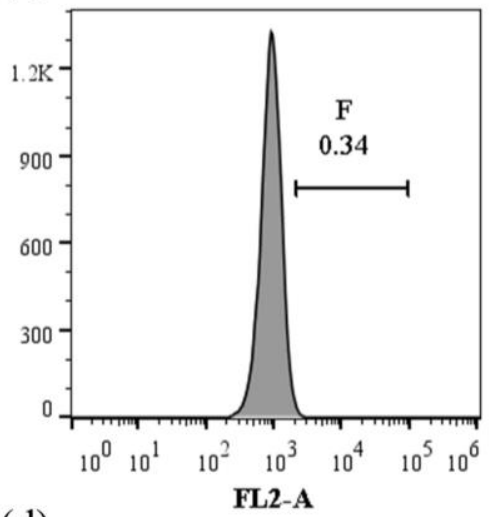

(d)

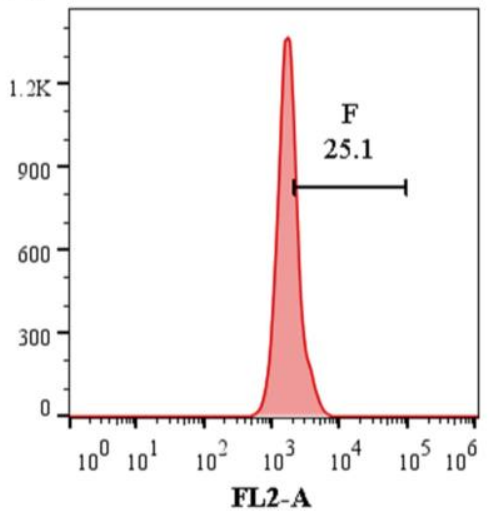

(b)

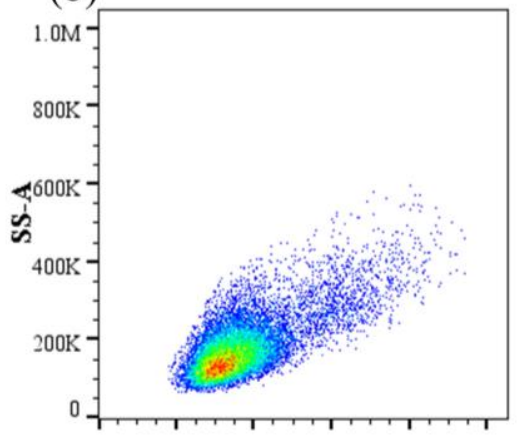

(e)

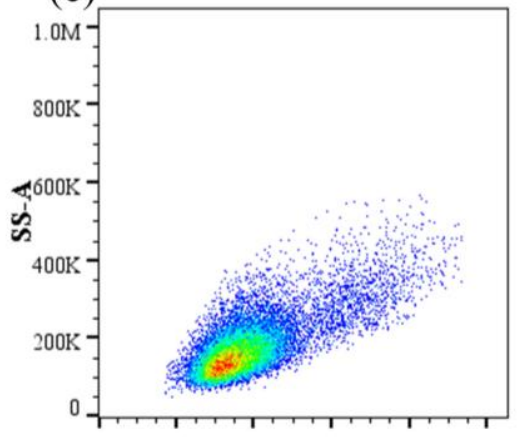

$\begin{array}{llllll}0 & 200 \mathrm{~K} & 400 \mathrm{~K} & 600 \mathrm{~K} & 800 \mathrm{~K} & 1.0 \mathrm{M}\end{array}$ (b) FS-A

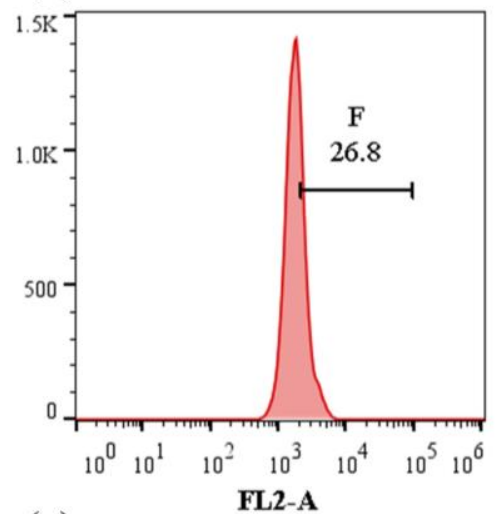

(e)

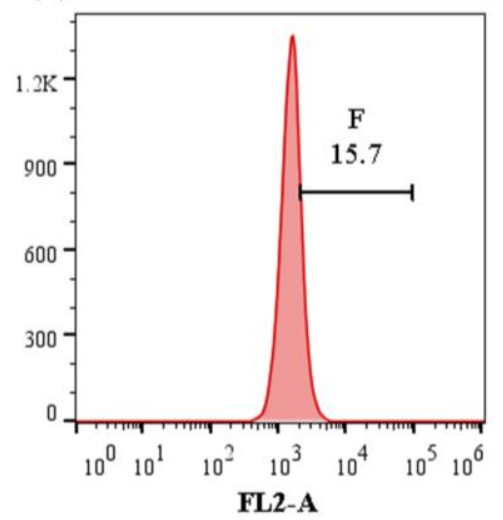

(c)

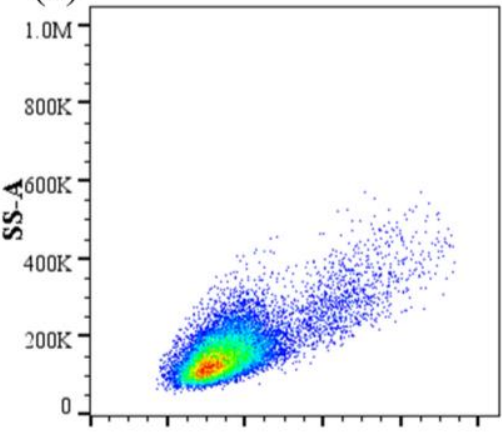

$\begin{array}{llllll}0 & 200 \mathrm{~K} & 400 \mathrm{~K} & 600 \mathrm{~K} & 800 \mathrm{~K} & 1.0 \mathrm{M}\end{array}$

(f) FS-A

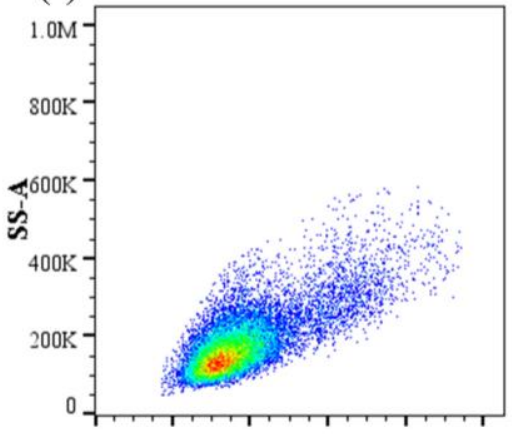

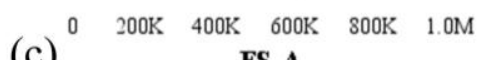
(c) FS-A

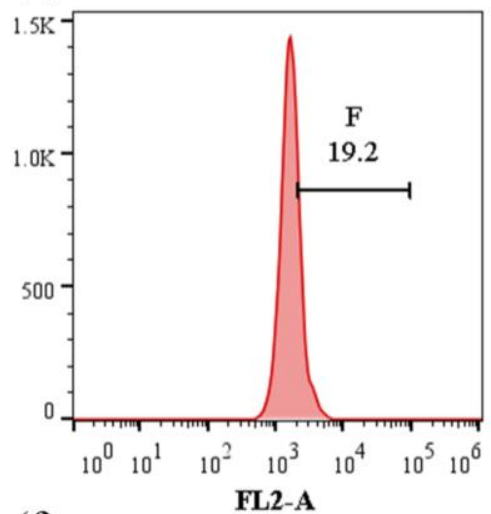

(f)

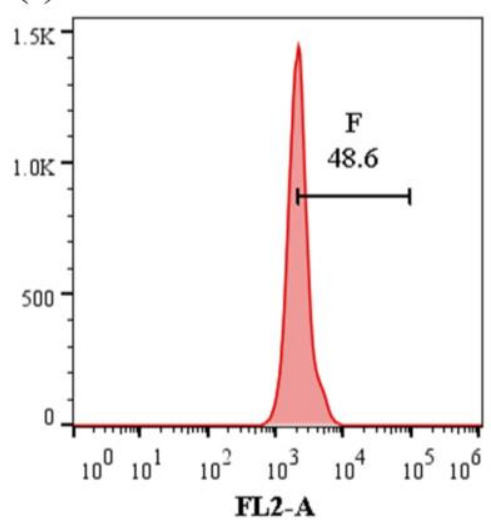

Figure S8. Representative images of surface markers CD163 of RAW264.7 analyzed by flow cytometry: (a) Blank; (b) Control; (c) Gel-MA; (d) Gel-MA/1\%NFB; (e) Gel-MA/3\%NFB; (f) Gel-M A/5\%NFB. 


\section{CCR7}

(a)

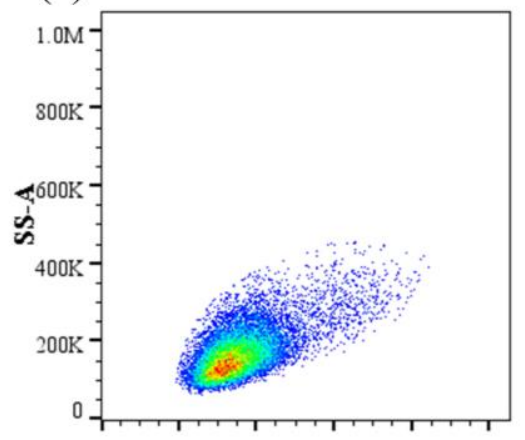

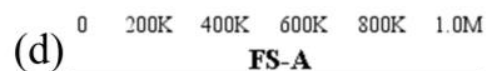

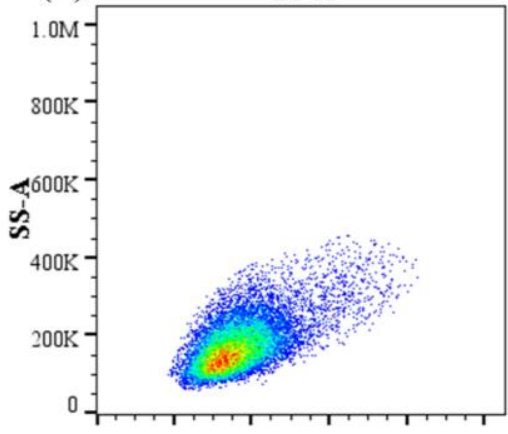

$\begin{array}{lllllll}0 & 200 \mathrm{~K} & 400 \mathrm{~K} & 600 \mathrm{~K} & 800 \mathrm{~K} & 1.0 \mathrm{M}\end{array}$

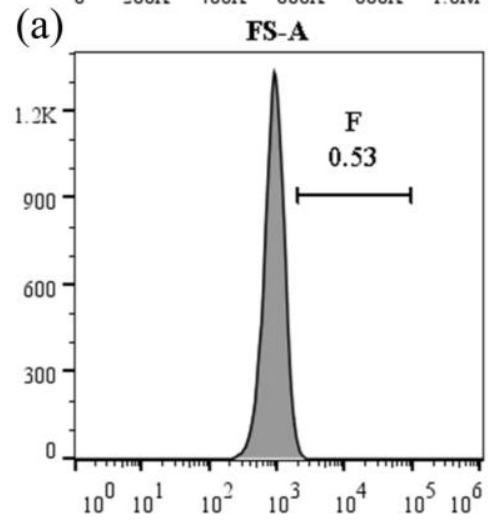

(d)

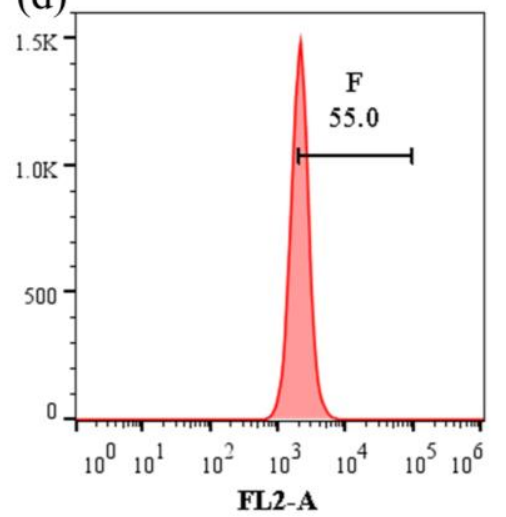

(b)

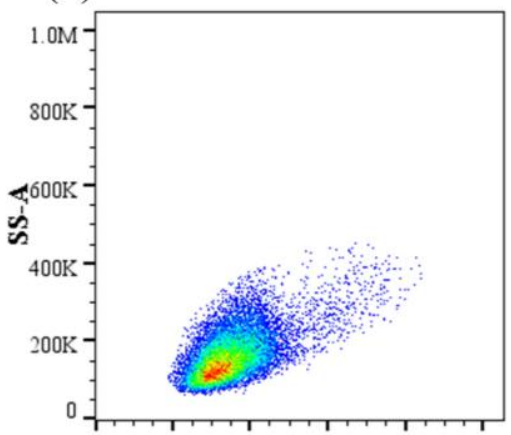

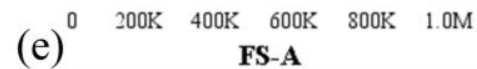

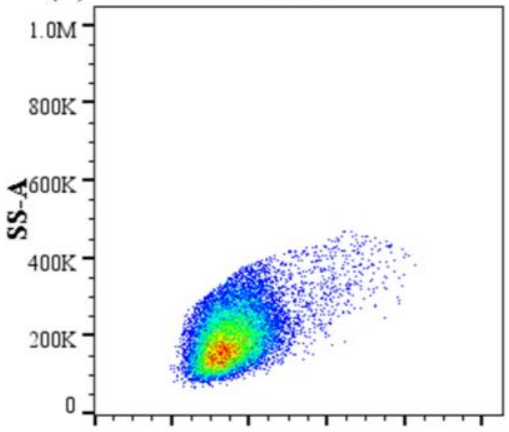

$\begin{array}{lllllll}0 & 200 \mathrm{~K} & 400 \mathrm{~K} & 600 \mathrm{~K} & 800 \mathrm{~K} & 1.0 \mathrm{M}\end{array}$ (b) FS-A

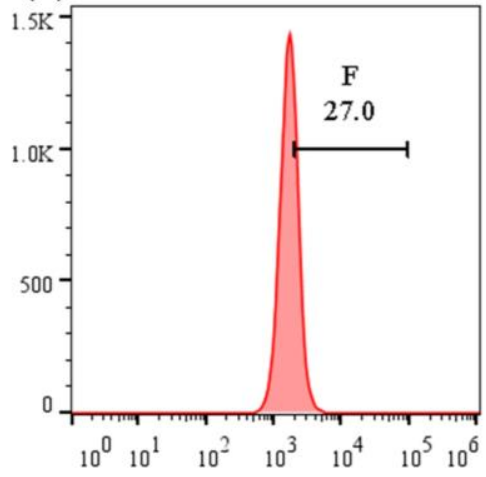

(e)

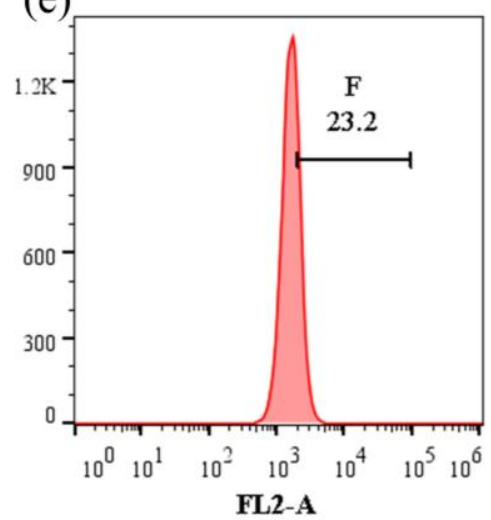

(c)

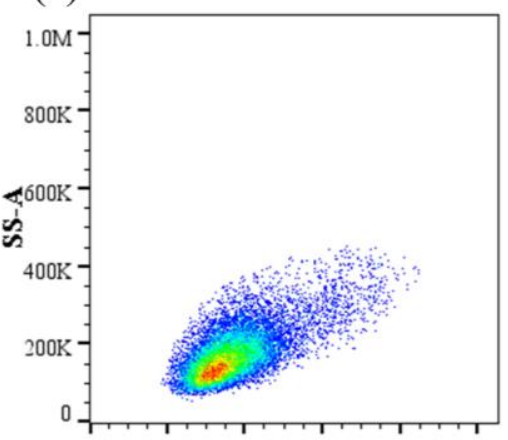

(f) $\begin{array}{llllll}0 & 200 \mathrm{~K} & 400 \mathrm{~K} & 600 \mathrm{~K} & 800 \mathrm{~K} & 1.0 \mathrm{M}\end{array}$
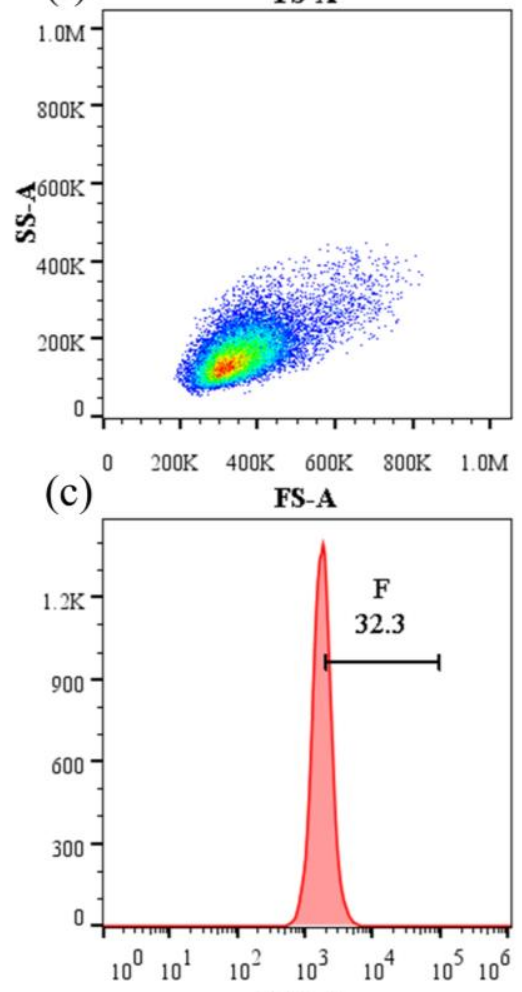

(f)

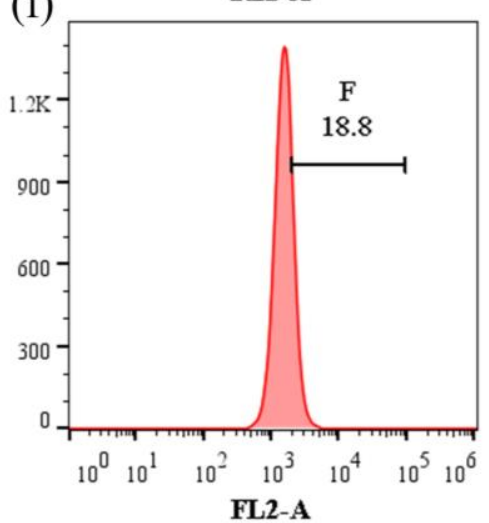

Figure S9. Representative images of surface markers CCR7 of RAW264.7 analyzed by flow cy tometry: (a) Blank; (b) Control; (c) Gel-M A; (d) Gel-MA/1\%NFB; (e) Gel-M A/3\%NFB; (f) Gel-M A/5\%NFB. 
(a)
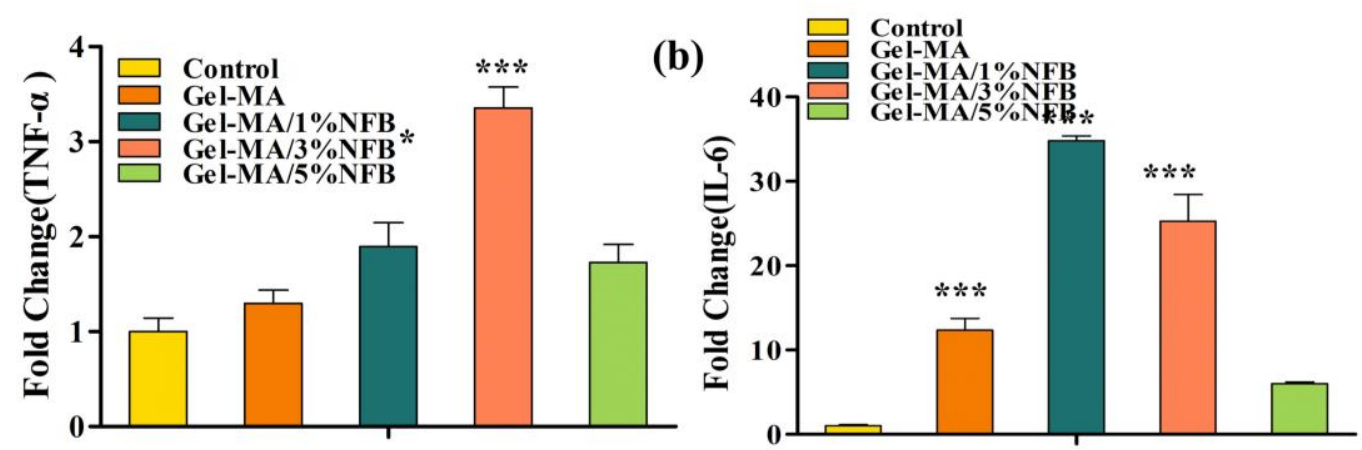

(c)

(d)

(e)
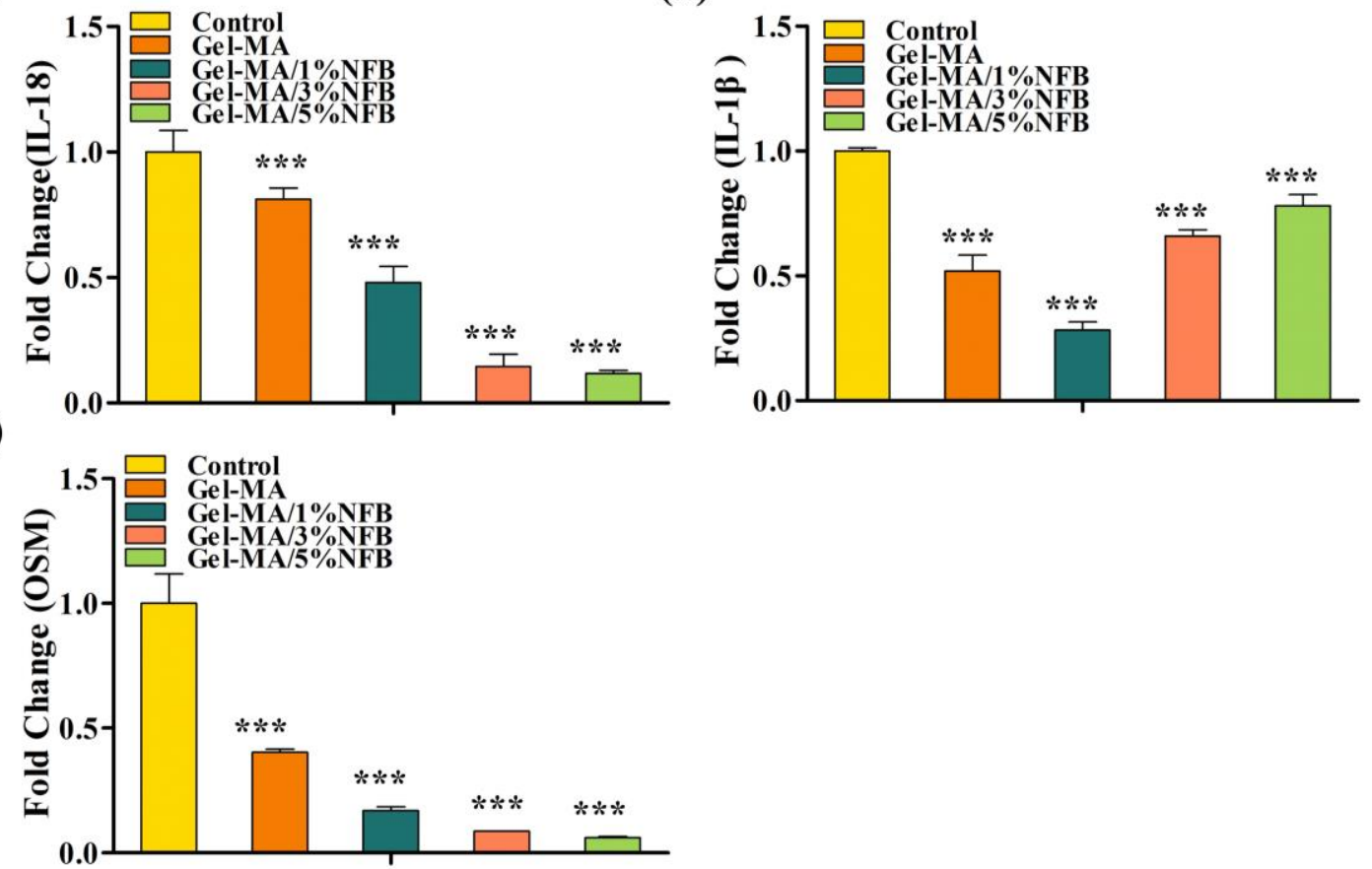

Figure S 10. Effects of Gel-M A hydrogels or Gel-M A/NFB hydrogels on the expression of inflammatory genes (TNF- $\alpha$, IL-6, IL-18, IL-1 $\beta$, OSM ) detected by RT-qPCR. The data were normalized to GADPH expression $\left({ }^{*} \mathrm{p}<0.05,{ }^{*} \mathrm{p}<0.01,{ }^{* * *} \mathrm{p}<0.001\right)$ as compared with the control group. 


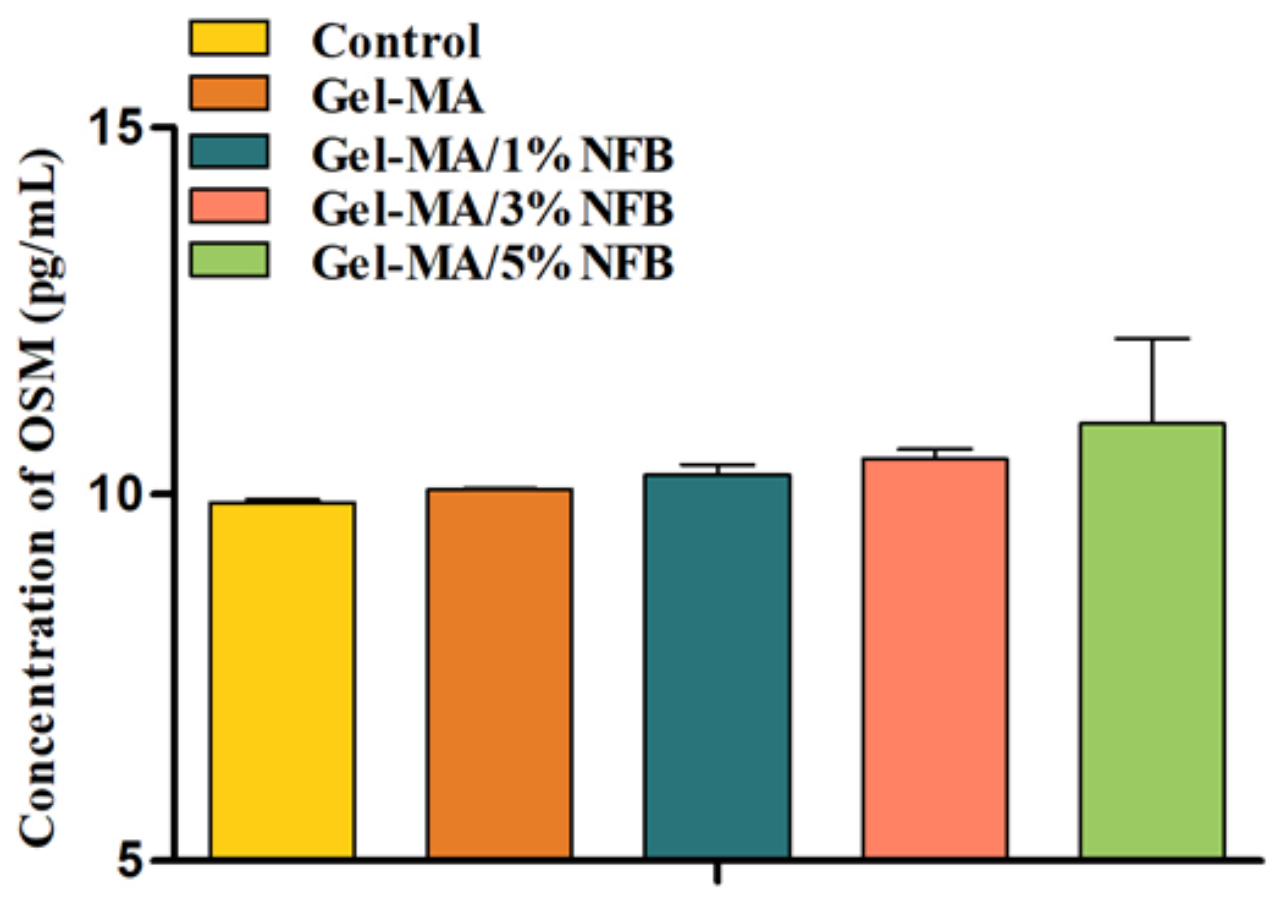

Figure S 11. Effects of Gel-M A hy drogels or Gel-M A/NFB hydrogels on the expression of inflammatory proteins (OSM) detected by ELISA. 


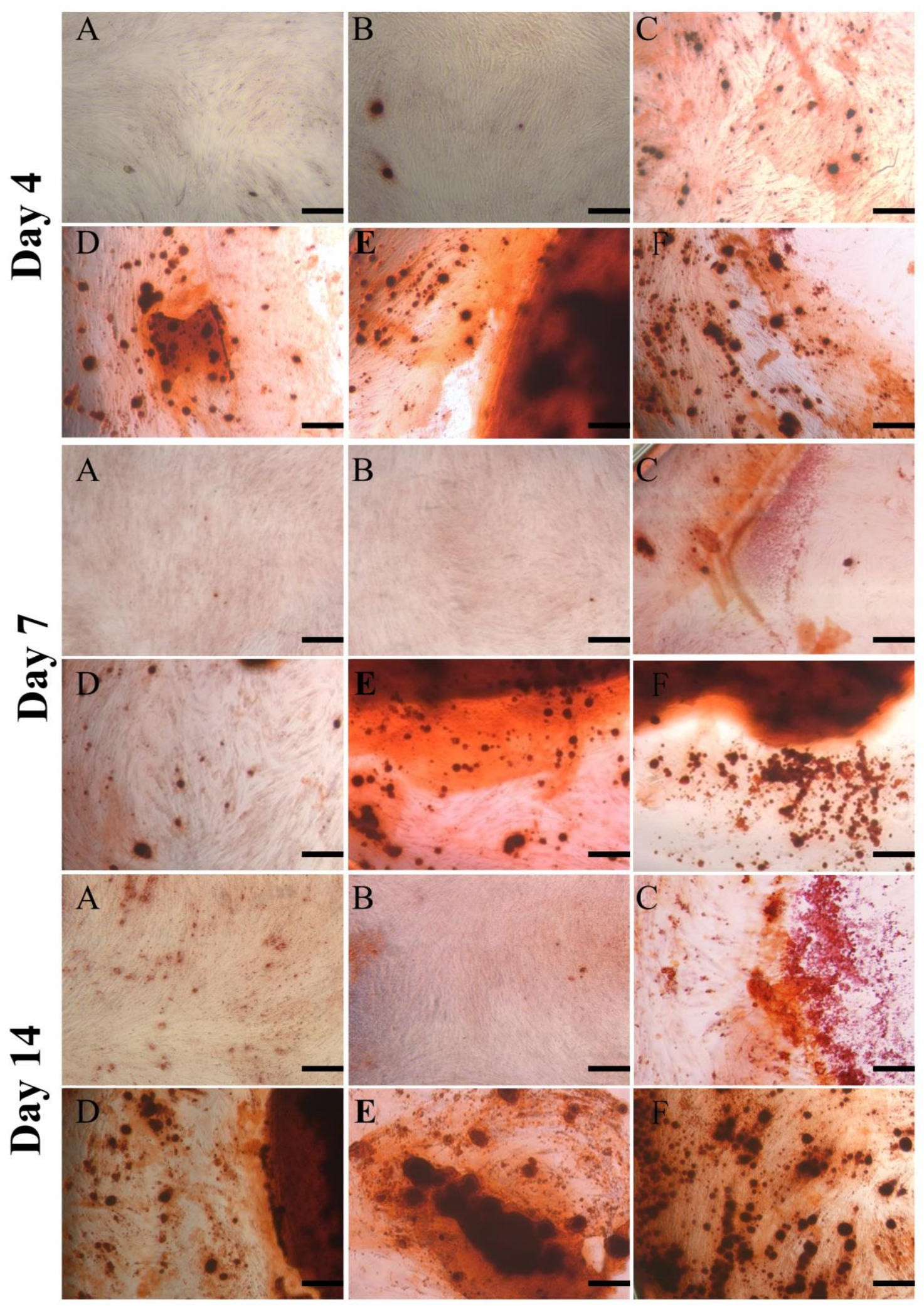

Figure S12. Alizarin red staining was used to demonstrate the mineralized nodules formed by hDPSCs' exposure to media conditioned by macrophages cultured on Gel-M A hydrogels or Gel-MA/NFB hydrogels. Scale bar = $100 \mu \mathrm{m}$. a) Blank; b) Control; c) Gel-MA; d) Gel-MA/1\%NFB; e) Gel-M A/3\%NFB;f) Gel-M A/5\%NFB. 


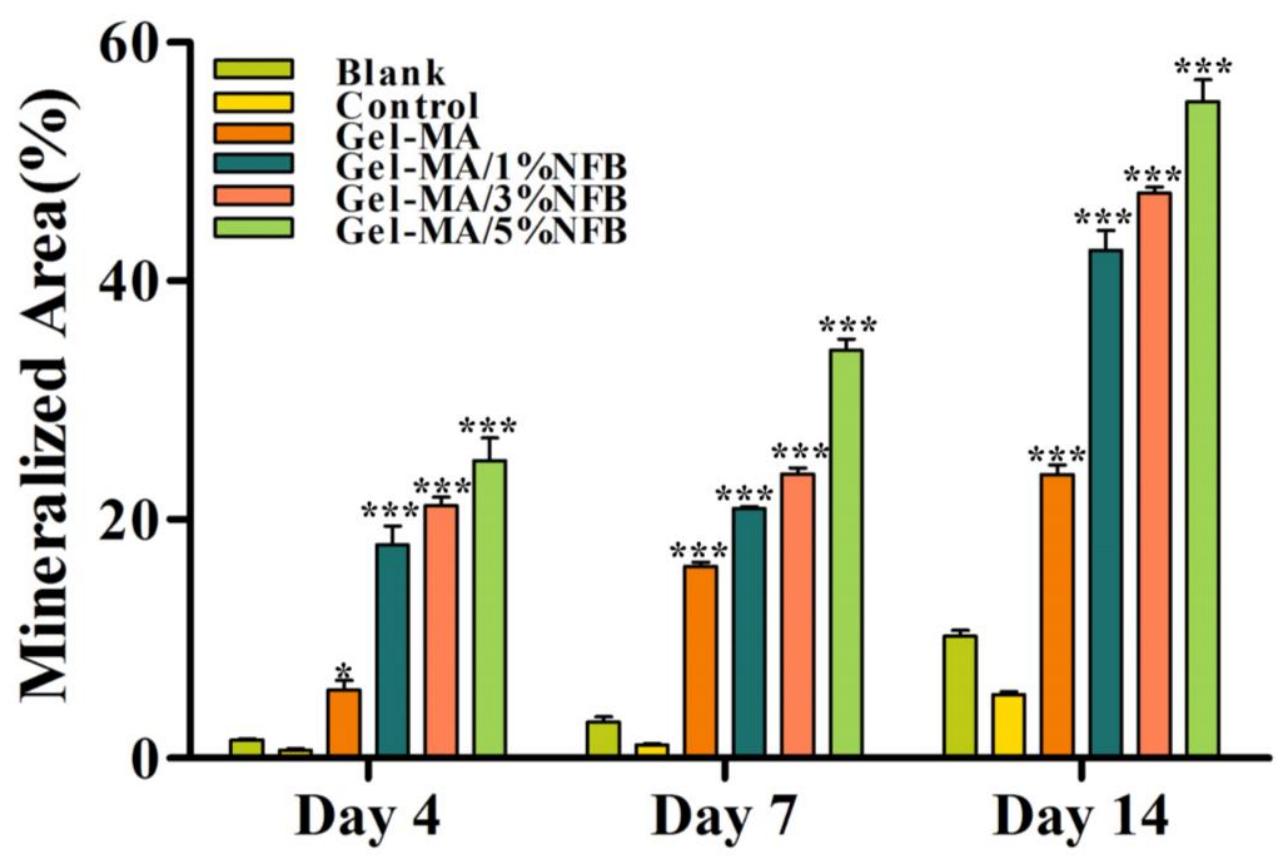

Figure S13. Image J analy sis of the mineralized area of a culture dish.
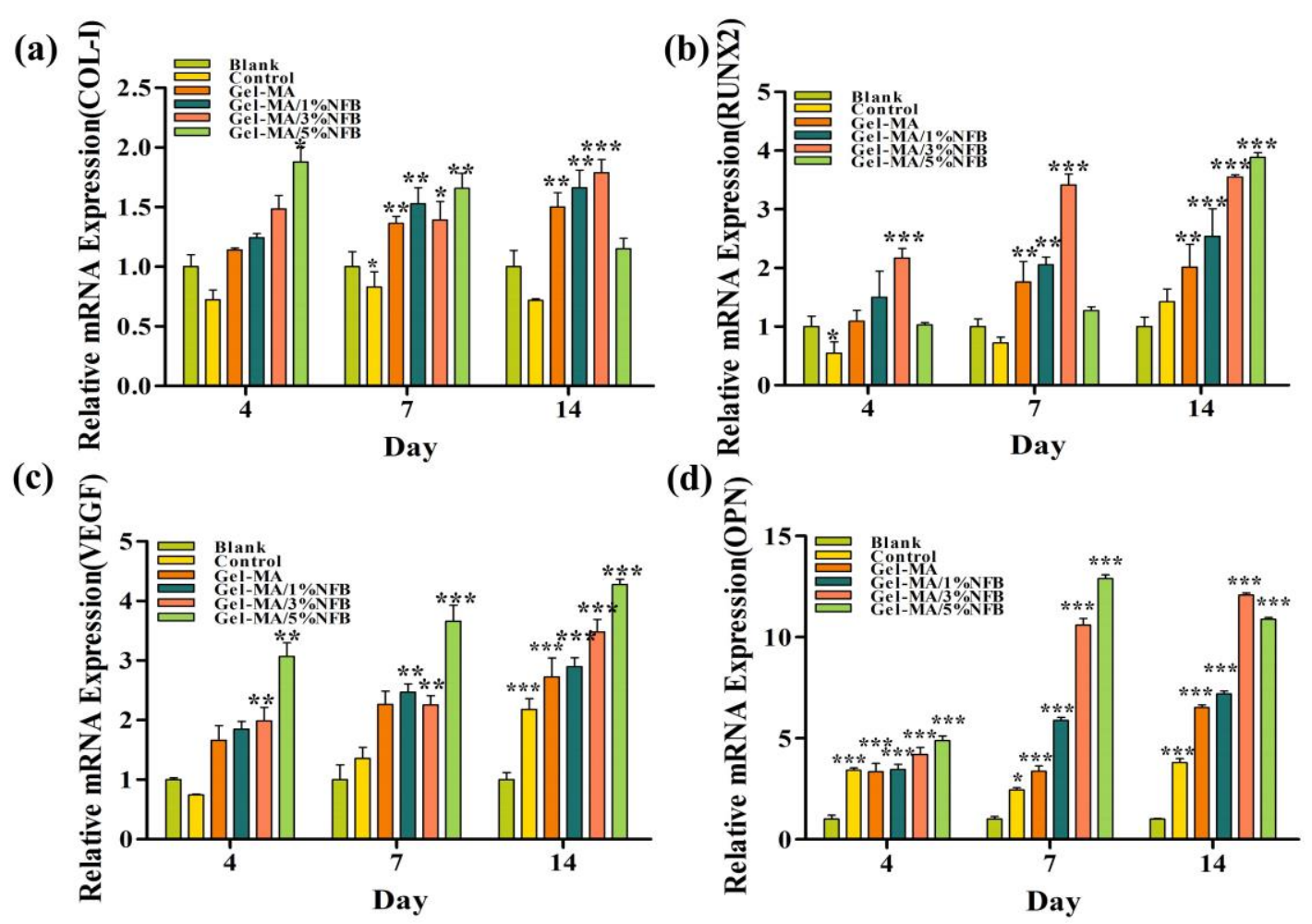
Figure S14. Effects of macrophage-conditioned medium of RAW264.7s osteogenic-related gene and angiogenic-related gene expression of hDPSCs after culturing for 4, 7, 14 day s (COL-I, RUNX-2, VEGF, OPN) detected by RT-qPCR. The data were normalized to GADPH expression $(* \mathrm{p}<0.05, * * \mathrm{p}<0.01, * * * \mathrm{p}<0.001)$ as compared with the blank group.
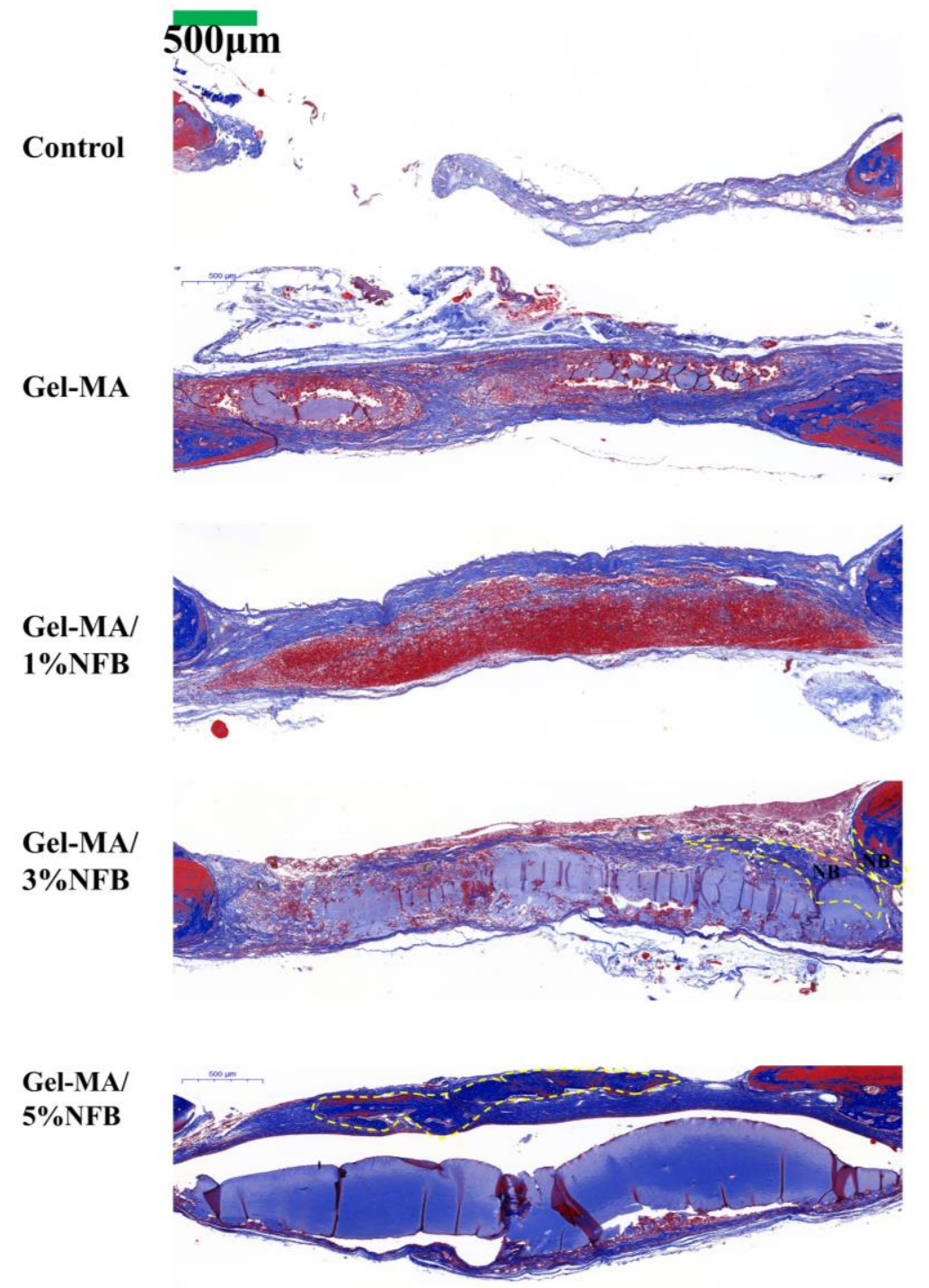

Figure S15. Masson staining of bone defect section at postoperative 4 weeks. 


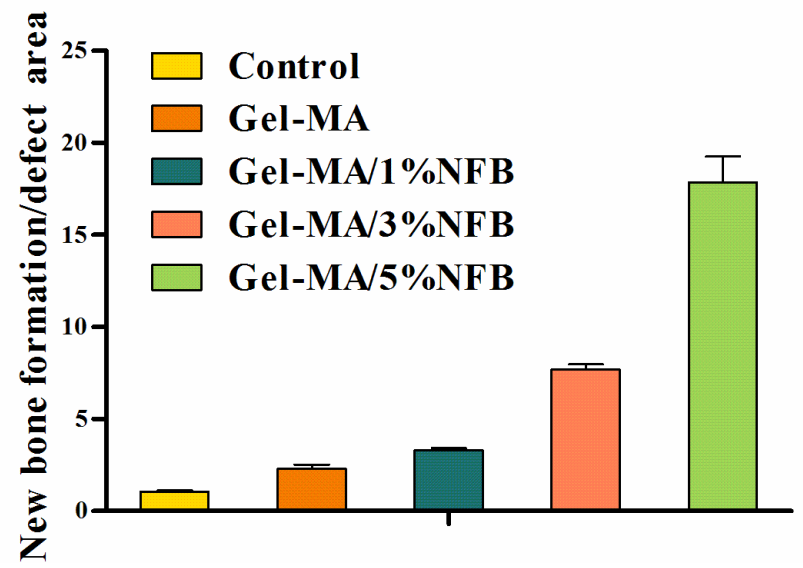

Figure S 16. Quantitative analy sis of H\&E staining with Image J.

(a)

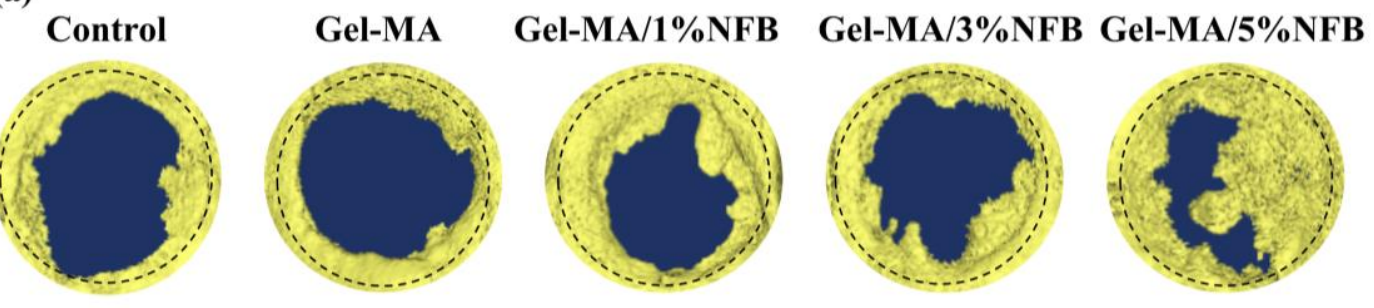

(b)

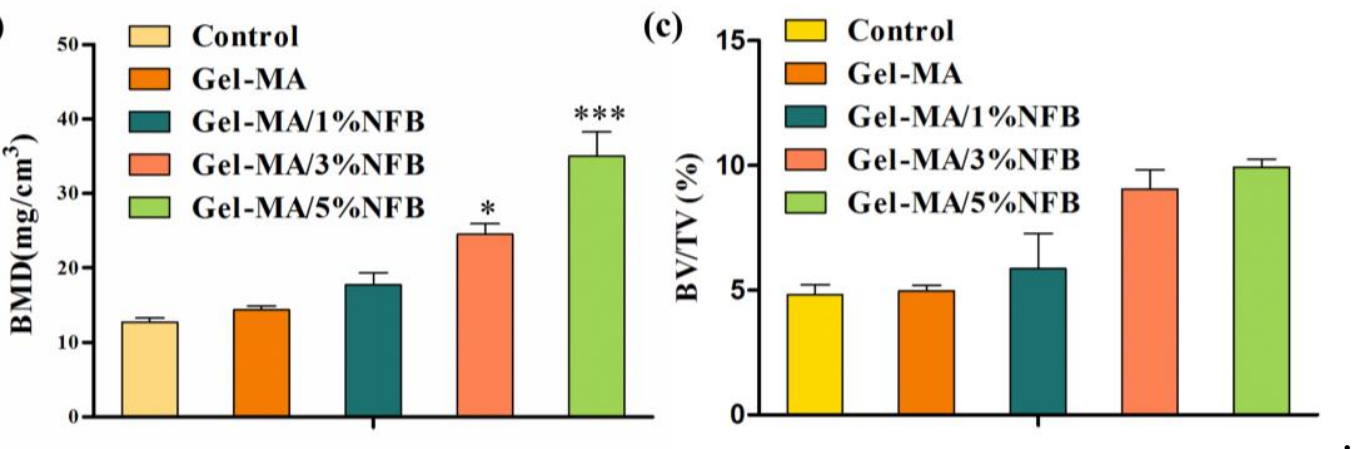

Figure S17. Micro-CT imaging and analy sis of harvested craniums obtained from Sprague-Dawley rats after treatments for 4 weeks.

(a) 3D reconstruction of micro-CT images of the cranium based on the density variations of osseous tissue. (b-c) Micro-CT quantification of the mineralized areas, as assessed by BMD, BV/TV. $(* \mathrm{p}<0.05$, **p $<0.01, * * * \mathrm{p}<0.001)$. 


\section{IL-1 $\beta$}

IL-6

IL-10

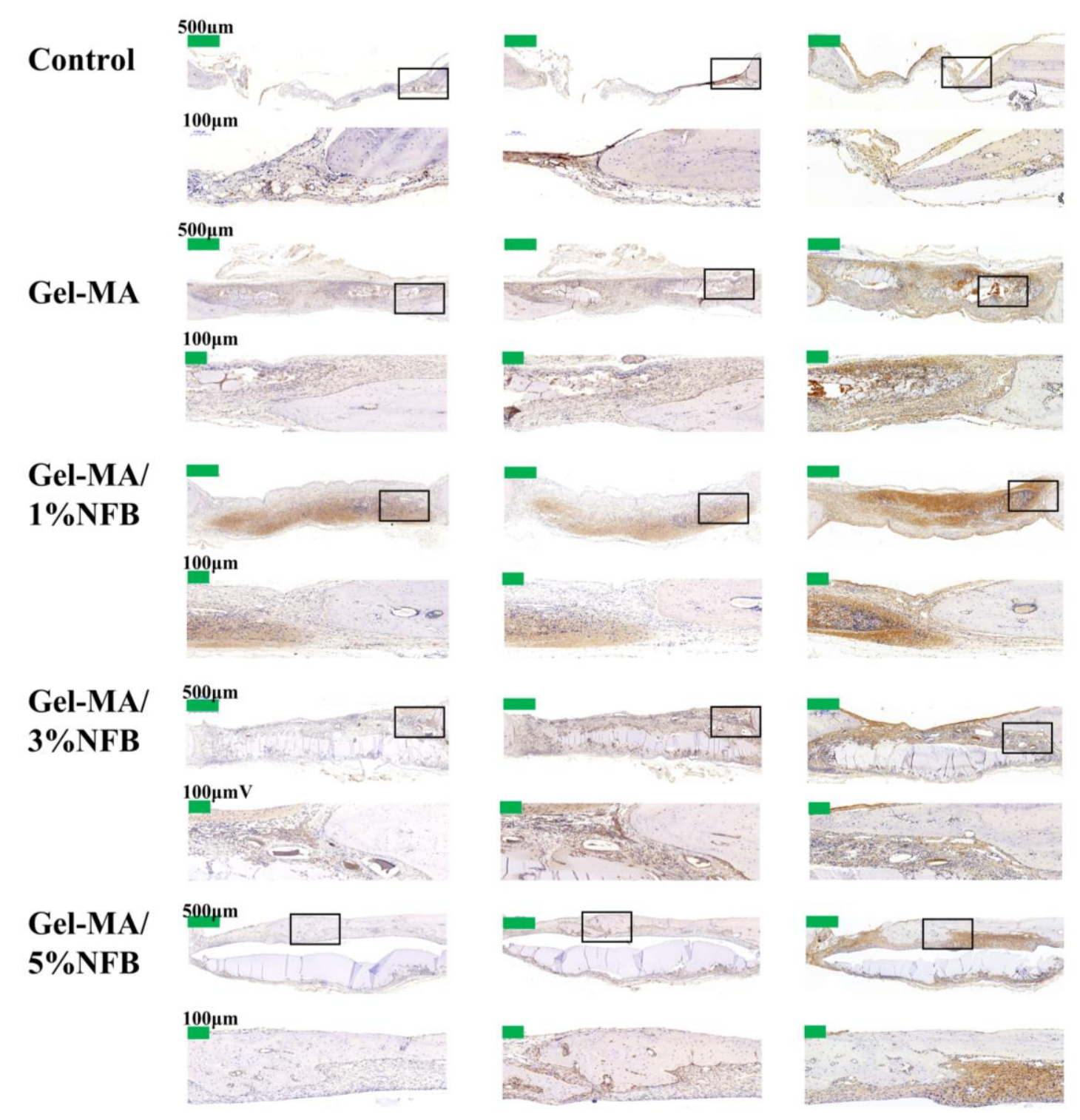

Figure S 18. Immunohistochemical staining of bone defect section at postoperative 4 weeks. 


\section{COL-I}

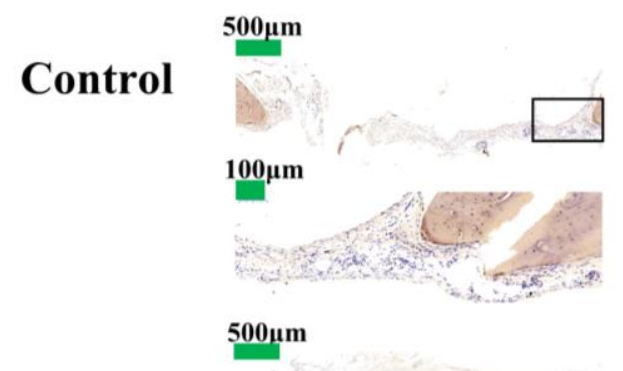

Gel-MA
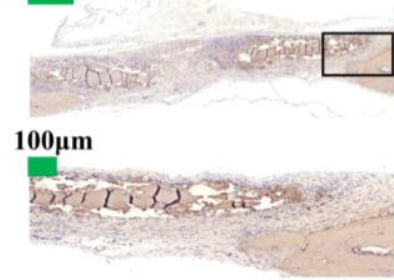

Gel-MA/

$$
500 \mu \mathrm{m}
$$

$1 \%$ NFB
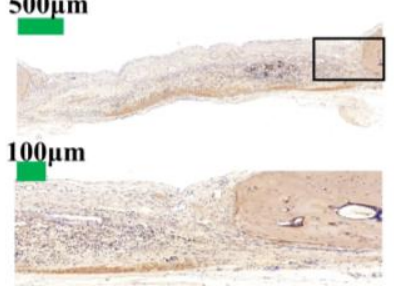

Gel-MA/ 3\%NFB

\section{$500 \mu \mathrm{m}$}
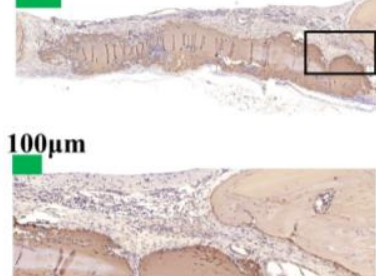

Gel-MA/ $500 \mu \mathrm{m}$ $5 \%$ NFB
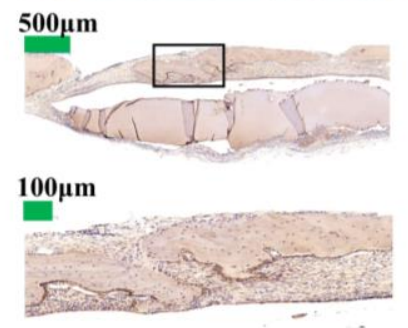

OPN
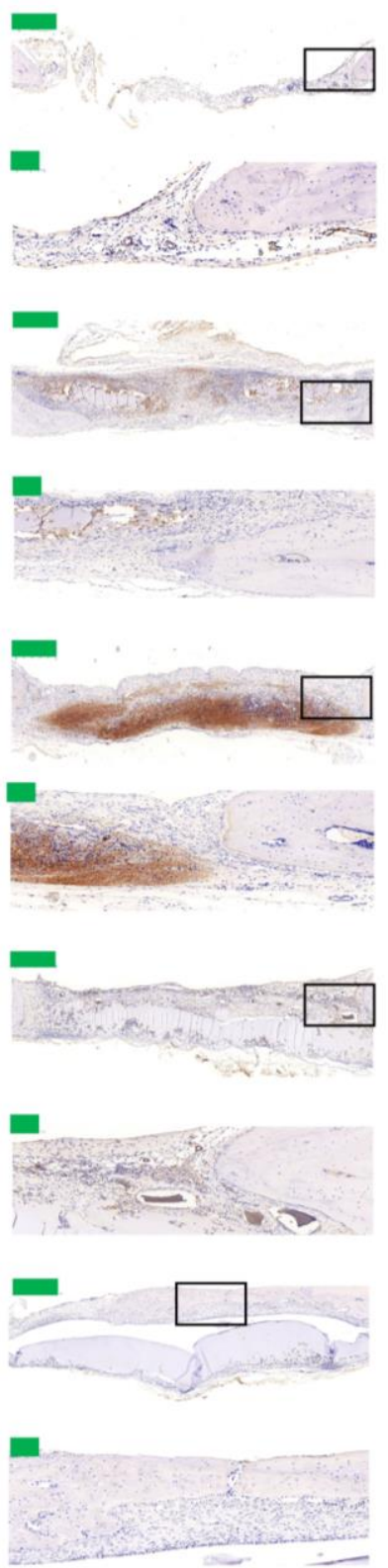

VEGF
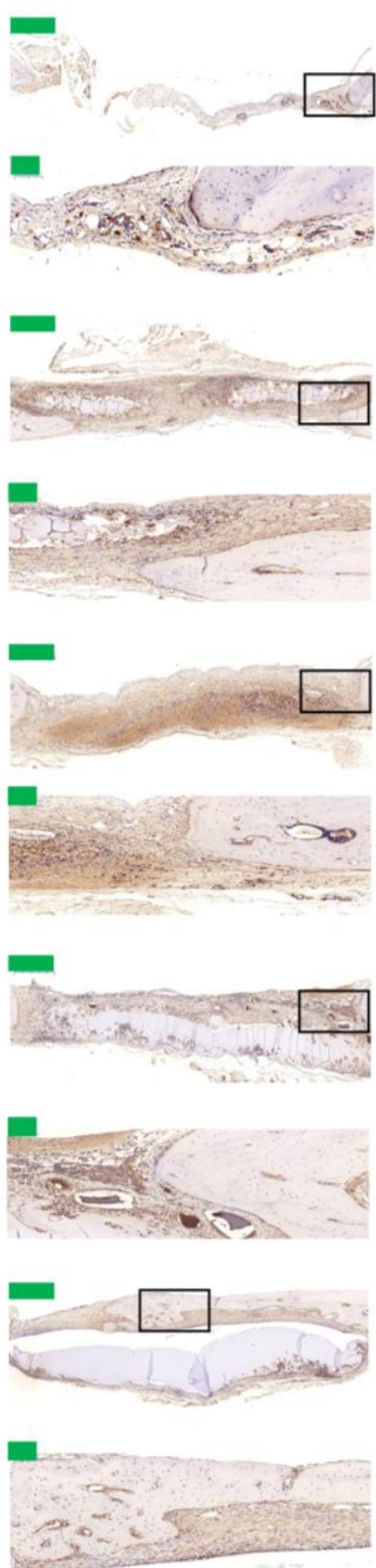

Figure S 19. The bone regeneration 4 weeks after surgery: Immunohistological staining of COL-1, OPN and VEGF for bone formation after 4 weeks.

\section{REFERENCES}

1. Yin, T.; Park, J. W.; Xiong, S. Phy sicochemical properties of nano fish bone prepared by wet media milling. LWT-Food. SCI. Technol. 2015, 64, 367-373, DOI: 10.1016/j.lwt.2015.06.007.

2. Seon Jeong Kim, S. J. P., Sun I. Kim. Welling behavior of interpenetrating polymer network hydrogels composed of poly (vinyl alcohol) and chitosan. React. Funct. Polym. 2003, 55, 53-59, DOI:10.1134/S0965545X11080062.

3. Ou, Q.; Wang, X.; Wang, Y.; Wang, Y.; Lin, X. Oestrogen retains human periodontal ligament stem cells stemness in long-term culture. Cell. Prolif. 2018, 51, 96-123, DOI: 10.1111/cpr.12396. 